\title{
Rotational bands beyond the Elliott model
}

\author{
Ryan Zbikowski, Calvin W. Johnson \\ Department of Physics, San Diego State University, San Diego, CA, 92182 \\ Computational Science Research Center, San Diego State University, San Diego, \\ CA, 92182
}

\author{
Anna E. McCoy \\ Institute for Nuclear Theory, University of Washington, Seattle, Washington \\ 98195-1550, USA \\ TRIUMF, Vancouver, British Columbia V6T 2A3, Canada
}

\section{Mark A. Caprio, Patrick J. Fasano}

Department of Physics, University of Notre Dame, Notre Dame, Indiana 46556-5670, USA

\begin{abstract}
Rotational bands are commonplace in the spectra of atomic nuclei. Inspired by early descriptions of these bands by quadrupole deformations of a liquid drop, Elliott constructed a discrete nucleon representations of SU(3) from fermionic creation and annihilation operators. Ever since, Elliott's model has been foundational to descriptions of rotation in nuclei. Later work, however, suggested the symplectic extension $\operatorname{Sp}(3, R)$ provides a more unified picture. We decompose no-core shell-model nuclear wave functions into symmetry-defined subspaces for several beryllium isotopes, as well as ${ }^{20} \mathrm{Ne}$, using the quadratic Casimirs of both Elliott's $\mathrm{SU}(3)$ and $\mathrm{Sp}(3, R)$. The band structure, delineated by strong $B(E 2)$ values, has a more consistent description in $\mathrm{Sp}(3, R)$ rather than $\mathrm{SU}(3)$. In particular, we confirm previous work finding in some nuclides strongly connected upper and lower bands with the same underlying symplectic structure.
\end{abstract}

Submitted to: J. Phys. G: Nucl. Part. Phys. 


\section{Introduction}

Among the earliest pictures of the atomic nucleus was its description as a tiny drop of dense fluid. Not only did this metaphor motivate the semi-empirical mass formula, it suggested interpreting nuclear excitation spectra as vibrations and rotations of such a drop [1]. While such a picture has many limitations, not least the lack of discrete nucleons, its consequences reverberate to this day.

To quantify the liquid drop model, the surface of the drop is parameterized in terms of spherical coordinates $\theta$ and $\phi$ and expanded in spherical harmonics: $R(\theta, \phi)=R_{0} \sum_{l, m} a_{l m} Y_{l m}(\theta, \phi)$. In the Bohr-Mottelson model, the coefficients $a_{l m}$ become dynamical variables. The $l=0$ term is usually a constant (if dynamic, it relates to breathing modes), and the $l=1$ terms are infinitesimal generators of displacement. Hence the quadrupole $l=2$ terms are the first, and often the last, important terms. The five degrees of freedom from $m=-2,-1,0,1,2$ correspond to the intrinsic shape deformation parameters $\beta, \gamma$ and three Euler angles for orientation [1-3]. The dynamics of the quadrupole deformation can be described by five mass quadrupole operators $\hat{Q}_{2 m}=r^{2} Y_{2 m}(\theta, \phi)$ plus the three components of (orbital) angular momentum $\mathbf{L}=\mathbf{r} \times \mathbf{p}$ [4]. Together these eight operators form generators of the rigid rotor group $\mathrm{ROT}(3)$ [5], or equivalently $\left[R^{5}\right] \mathrm{SO}(3)[6-8]$. This generator structure laid the foundation of describing nuclear spectral bands using group theory [9].

The Bohr-Mottelson model represents the nucleus as an undifferentiated fluid. Working under Giulio Racah, Phil Elliott [10] considered an alternative quadrupole operator $\hat{\mathcal{Q}}_{2 m}$, one which is equivalent to the mass quadrupole operator $\hat{Q}_{2 m}$ when restricted to a single harmonic oscillator single shell, but which does not connect different oscillator shells. These $\hat{\mathcal{Q}}_{2 m}$, taken together with the orbital angular momentum components $\hat{L}_{1 m}$, close under commutation. Elliott thereby arrived at a realization of the group $\mathrm{SU}(3)$ [11-15], generated by operators which conserve the number of oscillator quanta, which made it appropriate for phenomenological calculations with a frozen core and valence particles restricted to a limited single particle space such as a single oscillator shell $[11,15]$. For detailed discussions of the history and various models of rotation, see for example $[5,16]$.

While $\mathrm{SU}(3)$ is not the only algebraic framework describing rotational bandsindeed, it has been argued that the states of Elliott's SU(3) model are, under a technical definition, not rotational [4] except in a certain limit [16] — it is one of the best known and most widely referenced. A central question is whether an $\mathrm{SU}(3)$ picture is sufficient. Because Elliott's SU(3) model, by design, is restricted to states lying in the valence space, one can ask whether or not rotational bands extend to states outside the valence space [17-20]. A long-favored and natural alternative extension to $\mathrm{SU}(3)$ is the symplectic extension $\operatorname{Sp}(3, R)[5,21-24]$, which includes the kinetic energy and the mass quadrupole operator $\hat{Q}_{2 m}$ (which, unlike the quadrupole operator of Elliott's $\mathrm{SU}(3)$ model, is not restricted to a single shell).

In either an $\mathrm{SU}(3)$ or an $\mathrm{Sp}(3, R)$ rotational model, the rotational bands lie within irreducible representations (irreps), which are subspaces invariant under the generators of the respective group. Such a description of nuclear rotations is at best approximate, as realistic nuclear interactions break the invariance, mixing wavefunctions across irreps. Nonetheless, $\mathrm{SU}(3)$ and $\mathrm{Sp}(3, R)$ have been observed to be good approximate symmetries of nuclei [22-34] with the majority of the wavefunction spread over a relatively small number of irreps. Indeed, this is the motivation of calculations carried out in bases made up of irreps of a chosen algebra, for example $\mathrm{SU}(3)[27,28]$ or 
$\operatorname{Sp}(3 R)[32,34]$, which can be truncated to include only the most "important" irreps.

In this paper we carry out large-basis no-core shell model (NCSM) calculations of selected light nuclei using a realistic, microscopic interaction [35], and decompose their underlying algebraic structure, directly comparing $\mathrm{SU}(3)$ and $\operatorname{Sp}(3, R)$. It is important to emphasize that the NCSM calculations are done without any assumptions of underlying group structure. To examine the anatomy of rotational band members, which are recognized by strong $E 2$ transitions, we partition the wave functions into subspaces spanned by irreps with the same quantum numbers and find the fraction of each wave function contributed by each of these subspaces. We accomplish this by noting that these subspaces are eigenspaces of the Casimir operator of the relevant group, which allows us to use an efficient decomposition technique [36-38]. Rotational bands in NCSM calculations for the beryllium isotopes have been discussed in detail in references [20,39-42]. In this work, we focus on a selection of these $p$-shell nuclides $\left({ }^{7-10} \mathrm{Be}\right)$, as well as the $s d$-shell nucleus ${ }^{20} \mathrm{Ne}$.

We find that while both $\mathrm{SU}(3)$ and $\mathrm{Sp}(3, R)$ do provide an approximate descriptions of the rotational bands, $\operatorname{Sp}(3, R)$ provide a more consistent description than $\mathrm{SU}(3)$, in particular by including band members missed by $\mathrm{SU}(3)$. Furthermore, our work supports and extends a recent finding [34] that in some nuclei there exists an upper, excited band-like structure with strong $E 2$ transitions to the ground-state band, both bands sharing a common $\operatorname{Sp}(3, R)$ description. In fact, rather than being viewed as two separate but related bands, on the basis of our results we believe both "bands" should be considered part of a unified whole.

\section{Models and methods}

Physicists often refer to rotational and vibrational motion, but these are pictures taken from classical physics. Identifying analogous behavior in quantum systems, especially in complex many-body systems, is not trivial. One way to define a rotational band is as a set of states with distinct angular momentum quantum numbers, all projected from the same intrinsic state [1-3]. The wave function then factorizes into the product of a rotational wave function and an intrinsic wave function describing the structure in the body-fixed frame. (Such a body-fixed frame is only defined if the nucleus has quadrupole moments with small variances [4].) This assumption leads to specific predictions for ratios of electric quadrupole moments and $E 2$ transition strengths.

The most commonly observed case is of an axially symmetric instrinsic state. Here each band member has definite angular momentum $J$ and definite projection $K$ of $J$ onto the intrinsic symmetry axis. The projection $K$ is determined by the intrinsic state and is thus common to all band members, yielding a band consisting of states with angular momentum $J \geq K$. The energies of the band members are given by

$$
E(J)=E_{K}+A\left[J(J+1)-K^{2}+\delta_{K, \frac{1}{2}} a(-1)^{J+1 / 2}(J+1 / 2)\right],
$$

where the rotational energy constant $A \equiv \hbar^{2} /(2 \mathcal{I})$ is inversely proportional to the moment of inertia $\mathcal{I}$, and $E_{K}$ is the energy of the intrinsic state. The final term in brackets, which contributes only for $K=1 / 2$, arises from the Coriolis interaction and introduces an energy staggering between alternate band members.

Rotational bands are empirically confirmed through enhanced electric quadrupole transition strengths, or $B(E 2) \mathrm{s}$, among band members, where the overall scale of these is determined by the quadrupole moment of the intrinsic state [1-3]. While $E 2$ transitions may readily identify band members, the mere presence of rotational E2 
patterns provides no insight into the underlying intrinsic structure. Elliott's algebraic rotational model provided a first link between the collective rotational model and the microscopic shell model [11-15], but it is limited by the inherent assumption that the intrinsic state has definite $\mathrm{SU}(3)$ symmetry. In this work, we carry out our investigation in a shell model (or, more specifically, NCSM) framework, which makes no assumption of underlying symmetries. We review this shell model framework in section 2.1. Section 2.2 provides a brief introduction to the groups and their associated algebras which underpin the dynamical symmetries we are looking for in the nuclear system. To investigate the symmetries of members of our calculated rotational bands, we decompose them by partitioning the wave functions into subspaces defined by the symmetry, as described in section 2.3.

\subsection{The shell model context}

We carry out our investigation in the context of the configuration-interaction method: the nuclear wave function is expanded in a basis,

$$
|\Psi\rangle=\sum_{\alpha} v_{\alpha}|\alpha\rangle,
$$

where the basis states are, for example, antisymmetrized products of single-particle states or Slater determinants, or their representation in occupation space, $|\alpha\rangle=$ $\hat{a}_{1}^{\dagger} \hat{a}_{2}^{\dagger} \hat{a}_{3}^{\dagger} \ldots \hat{a}_{A}^{\dagger}|0\rangle$. Such basis states have a discrete, finite number of fermions, and with a sufficiently large basis one can in principle describe any nucleus [43-45].

In configuration-interaction calculations one uses single particle states with good angular momentum, that is, eigenstates of the total squared angular momentum operator $\hat{J}^{2}$ and $z$-component $\hat{J}_{z}$. Often one uses a harmonic oscillator single particle basis, in part because matrix elements of translationally invariant operators, including the Hamiltonian, are then straightforward to compute, and in part because this also allows one to rigorously address center-of-mass motion [46], concerns peripheral to our work here. The single-particle harmonic oscillator Hamiltonian $\hat{H}_{0}=-\frac{\hbar^{2}}{2 m} \nabla^{2}+$ $\frac{1}{2} m \omega^{2} r^{2}$ has eigenvalues $\hbar \omega(N+3 / 2)$, where $N \equiv 2 n+l$ is the principal quantum number, in terms of the orbital angular momentum $l$ and the number of nodes $n$ in the radial wave function. In common usage the distinction between orbitals and shells can be vague. We define orbitals as states with a specified $n, l$, and $j$, while shells (or major oscillator shells) are defined by $N$.

In empirical many-body spaces, such as those addressed originally by the Elliott model, one has a frozen, inert core, with all orbitals occupied up to some maximum, and the valence or active space is a fixed set of orbitals, often a major oscillator shell, such as the $p$-, $s d$ - or $p f$-shells.

In contrast, no-core or ab initio shell model (NCSM) calculations allow particles to be excited in and out of many shells $[47,48]$. The model space is usually specified as follows: for any Slater determinant (or its occupation-space representation) with fixed numbers of protons and neutrons, let $N_{A}=\sum_{i} N_{i}$, where $N_{i}$ is the principal quantum number for the $i$ th particle. That is, $N_{A}$ is the total number of oscillator quanta in the many-body state, and, when applied to an $A$-body state, the eigenvalue of $\hat{H}_{0}$ is $\hbar \omega\left(N_{A}+3 A / 2\right)$. There will be some minimum value of $N_{A}, N_{0}$, dictated by the Pauli principle. One often labels configurations not by their absolute $N_{A}$ but rather by the number of excitation quanta $N_{\mathrm{ex}}=N_{A}-N_{0}$ above the minimum. These configurations are also called $N_{\text {ex }} \hbar \omega$ excitations (i.e., $0 \hbar \omega, 2 \hbar \omega$, etc.), in reference to the corresponding oscillator energy. 
The truncation of the no-core shell model many-body space is specified by $N_{\text {max }}$, which is the maximal $N_{\text {ex }}$ for the basis configurations (that is, the difference between the minimal and maximal allowed values of $N_{A}$ ). To fully define the space, one must also specify the frequency of the single-particle harmonic oscillator basis, conventionally quoted as the oscillator energy $\hbar \omega$. Because the parity for a single-particle orbital is $\pi=(-1)^{l}=(-1)^{N}$, configurations with even $N_{\text {ex }}$ $\left(N_{\mathrm{ex}}=0,2,4, \ldots\right)$ have "natural" parity (i.e., that of the lowest Pauli allowed filling), while configurations with odd $N_{\mathrm{ex}}\left(N_{\mathrm{ex}}=1,3,5, \ldots\right)$ have the opposite, "unnatural" parity.

The $N_{\max }=0$ space, consisting of configurations with $N_{\mathrm{ex}}=0$, corresponds to the typical valence model space of empirical shell model calculations carried out in a single oscillator shell. Here, in order to connect our multi-shell calculations with such empirical calculations, we will often refer to the $N_{\text {ex }}=0$ subspace as the valence subspace. For example, in the beryllium isotopes we consider below, the valence space consists of states with filled $0 s_{1 / 2}$ orbital (that is, a ${ }^{4} \mathrm{He}$ core) with valence protons and neutrons restricted to the $0 p$ shell, while for ${ }^{20} \mathrm{Ne}$ the valence space has a ${ }^{16} \mathrm{O}$ core with valence nucleons in the $1 s-0 d$ shell. Most low-lying states in NCSM calculations have a dominant fraction of the wave function in the valence subspace.

NCSM calculations use realistic nucleon-nucleon interactions, by which we mean interactions fitted primarily to two- and three-body data such as scattering phase shifts and deuteron properties, which are then applied to $A$-body systems such as we describe herein. Here we used a chiral next-to-next-to-next-to-leading order (N3LO) interaction [35]. We evolved the interaction via the similarity renormalization group [49] to a standard value of $\lambda=2.0 \mathrm{fm}^{-1}$. We did not use three-body forces, although their effect would be interesting in future work.

For the many-body calculation we used the BIGSTICK code [50,51], which solves the large, sparse, many-body eigenvalue problem via the Lanczos algorithm [52], and which uses an $M$-scheme basis, that is, the many-body basis fixes the total $M$ (or eigenvalue of or $\hat{J}_{z}$ ), and the overall parity, but no other quantum numbers. We carried out calculations of ${ }^{7} \mathrm{Be}$ and ${ }^{8} \mathrm{Be}$ at $N_{\max }=10$, while the 'unnatural' parity states of ${ }^{9} \mathrm{Be}$ we computed at $N_{\max }=9$, and ${ }^{10} \mathrm{Be}$ we computed in a $N_{\max }=8$ space. Finally, we studied ${ }^{20} \mathrm{Ne}$ in an $N_{\max }=4$ space. These calculations had $M$-scheme basis dimensions between a few tens of millions and a few hundreds of millions. All of our calculations used a harmonic oscillator basis frequency of $\hbar \omega=20 \mathrm{MeV}$.

Our guiding example in this paper is ${ }^{7} \mathrm{Be}$. Fig. 1 plots the excitation energies of low-lying negative (natural) parity states of ${ }^{7} \mathrm{Be}$, versus angular momentum $J$, with $J$ spaced as $J(J+1)$, as appropriate for rotational analysis. We also plot the downward ( $J$-decreasing) $B(E 2)$ strengths, computed using the standard operator [43] with bare charges, as solid lines, with the width and shading approximately proportional to the $\log$ strength. The weakest transitions are omitted, for visual clarity. NCSM $B(E 2)$ values are known to be sensitive both to the choice of $N_{\max }$ and of the oscillator parameter $\hbar \omega$ for the single-particle wave functions. Nonetheless, the relative strengths of the $B(E 2)$ values are comparatively insensitive to these choices and are therefore useful in identifying members of a band. (See, e.g., references $[41,42]$ for convergence studies of NCSM calculations for the nuclei considered here.) For example, here for ${ }^{7} \mathrm{Be}$, from the relative $E 2$ strengths in Fig. 1 it can be seen that, while most of the yrast states belong to a clearly identified $K=1 / 2$ ground-state band, the yrast $9 / 2_{1}^{-}$is not part of the ground-state band; rather it is the $9 / 2_{2}^{-}$state that is strongly connected to the band. 


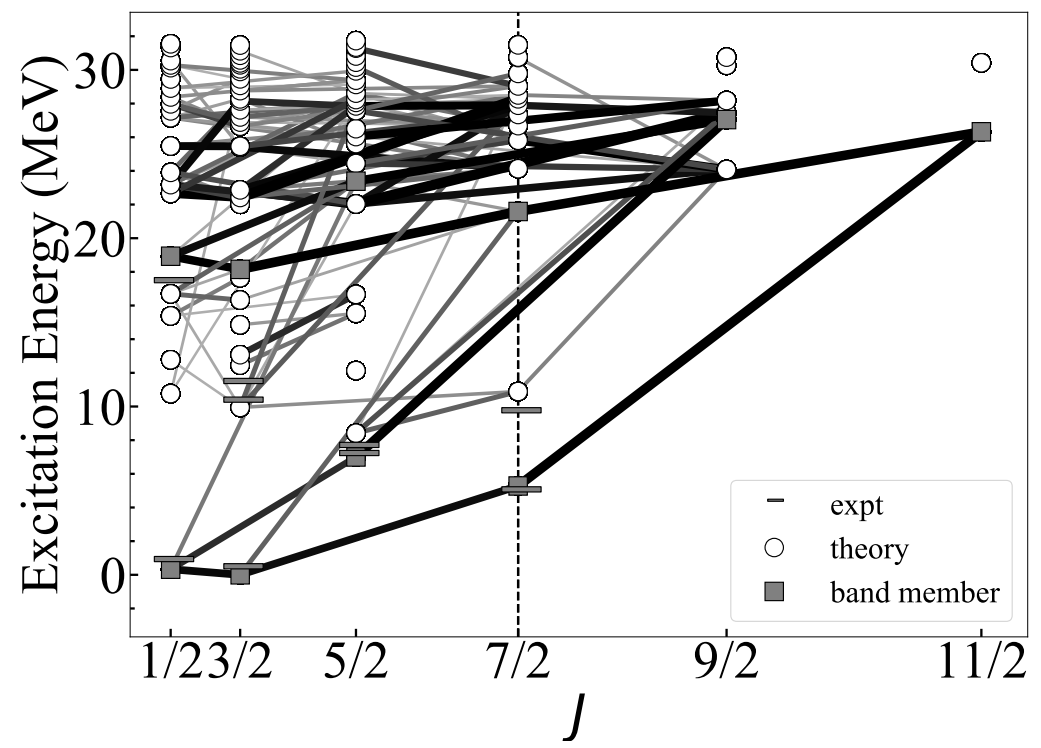

Figure 1: Excitation spectrum of the natural, negative parity states of ${ }^{7} \mathrm{Be}$, computed at $N_{\max }=10$ with a single-particle harmonic oscillator basis frequency $\hbar \omega=20 \mathrm{MeV}$, using a two-body chiral force at N3LO. Experimental energies are presented as shaded bars are shown for comparison (see text). Rotational band members are indicated using shaded squares. These states are decomposed into group irreps in Figs. 2 and 3. 'Downward' (specifically from higher $J$ to lower $J$ ) $E 2$ transitions connecting to band members are indicated with solid lines. Line thickness and shading is proportional to $B(E 2)$ strength. Only strengths above $1.5 e^{2} \mathrm{fm}^{4}$ are shown. The vertical dashed line denotes the maximal angular momentum within the lowest harmonic oscillator configuration, that is, the valence subspace.

\subsection{Dynamical symmetries and group theoretical framework}

Symmetries and, in particular, dynamical symmetries provide valuable insight into the nature of nuclear states and the nuclear excitation spectrum [8,9,53-57]. Dynamical symmetries arise by construction in algebraic models [8], which provide a simplified description of nuclear dynamics, yet they can persist in the full nuclear many-body problem with realistic nuclear forces.

Most familiar, perhaps, is the case in which the Hamiltonian is invariant under a set of transformations that define the symmetry group [8] for the problem. For example, the rotationally invariant nuclear many-body problem has as its symmetry group $\mathrm{SO}(3)$ or, since fermions are involved, $\mathrm{SU}(2)$. If the Hamiltonian is invariant with respect to symmetry transformations, then the symmetry group can only transform an eigenstate into other degnerate eigenstates, thereby forming a degnerate multiplet. Thus it is convenient to classify eigenstates into irreducible representations (irreps) of the symmetry group. Irreps are subspaces (of the full Hilbert space) which are invariant under the group transformations and which cannot be reduced further, i.e., broken down into smaller invariant subspaces. 
The irreps of a symmetry group are labeled by a quantum number or numbers $\Gamma$. The states within an irrep can be labeled by some additional quantum number(s) $i$, as $|\Gamma i\rangle[54]$. For the familiar case of $\mathrm{SU}(2)$, the irreps are the angular momentum multiplets $|J M\rangle$.

Often many irreps in a Hilbert space share the same $\Gamma$, requiring additional quantum numbers denoted generically by $\alpha[8,9,54]$, so that the states are $|\alpha \Gamma i\rangle$. For instance, the space for a rotationally invariant problem will contain many states of the same angular momentum $J$, and the eigenstates are fully labeled as $|\alpha J M\rangle$.

While states naturally form irreps when the Hamiltonian is invariant under a symmetry group, they may also be organized into irreps in the more general case of dynamical symmetry. A simple, classic example of dynamical symmetry, relevant to the present investigation, can be constructed in Elliott's SU(3) model [11-15] with the right choice of interaction within the nuclear shell model.

Elliot's SU(3) group was constructed with deformation and rotation in mind, and with generators chosen so that the group transformations do not excite nucleons between major shells. The generators include special quadrupole operators

$$
\hat{\mathcal{Q}}_{2 m}=\sum_{i=1}^{A} \sqrt{\frac{4 \pi}{5}}\left(\frac{r_{i}^{2}}{b^{2}} Y_{2 m}\left(\hat{\mathbf{r}}_{i}\right)+b^{2} p_{i}^{2} Y_{2 m}\left(\hat{\mathbf{p}}_{i}\right)\right),
$$

where $b$ is the shell model oscillator length parameter. Unlike the usual (mass) quadrupole operators $\hat{Q}_{2 m}$, the Elliott quadrupole operators $\hat{\mathcal{Q}}_{2 m}$ conserve the number of oscillator quanta; yet within a single shell they are simply proportional to the usual $\hat{Q}_{2 m}$. The remaining generators are the orbital angular momentum operators $\hat{L}_{1 m}=\sum_{i} \hat{L}_{1 m, i}$, which by themselves generate the group $\mathrm{SO}(3)$ of rotations on the coordinate degrees of freedom. Elliott chose the definition (3) so as to ensure that the $\hat{\mathcal{Q}}_{2}$ and $\hat{L}_{1}$ operators close under commutation, and have the commutator structure of $\mathrm{SU}(3)$ group generators. We thus have a subgroup chain $\mathrm{SU}(3) \supset \mathrm{SO}(3)$.

In Elliott's SU(3) framework a natural shell model Hamiltonian is [15]

$$
H=-\chi \mathcal{Q} \cdot \mathcal{Q},
$$

where the dot represents the standard spherical tensor scalar product [9]. In this model, rotational bands emerge from $\mathrm{SU}(3) \supset \mathrm{SO}(3)$ irreps. An irrep of $\mathrm{SU}(3)$ is labeled by two quantum numbers $(\lambda, \mu)$. Depending on the values of $(\lambda, \mu)$, the $\mathrm{SU}(3)$ irrep will contain several $\mathrm{SO}(3)$ irreps, that is, states having different values $L$ of the total orbital angular momentum. These states naturally organize into one or more rotational bands [15], e.g., a $(4,2)$ irrep contains states with $L=0,2,4(K=0)$ and $L=2,3,4,5,6(K=2)$. The total orbital angular momentum $L$ and the total spin $S$ combine to a total angular momentum $J$. The simple Elliott Hamiltonian (4) gives bands with energies depending only on the orbital angular momentum, as $L(L+1)$, but Elliott and Wilsdon [14] showed the microscopic spin-orbit interaction mixes states of different $L$ (but the same $J$ ) to give rotational bands with the familiar rotational dependence (1) of energies on $J$.

A natural extension of Elliott's $\mathrm{SU}(3)$ group is the symplectic group $\operatorname{Sp}(3, R)[5$, 21-24] in three dimensions. In addition to the $\mathrm{SU}(3)$ generators $\hat{\mathcal{Q}}_{2 m}$ and $\hat{L}_{1 m}$, the generators of this group include the harmonic oscillator Hamiltonian $\hat{H}_{0}$ and symplectic raising and lowering (or ladder) operators $\hat{A}_{l m}$ and $\hat{B}_{l m}$, with $l=0$ and 2. These ladder operators physically represent creation and annihilation operators, respectively, for giant monopole and quadrupole resonances. Unlike the $\mathrm{SU}(3)$ generators, which conserve the number of oscillator quanta, the symplectic raising 
and lowering operators add or remove two quanta, respectively, to the nuclear manybody state. They thus connect $N_{\text {ex }} \hbar \omega$ shell model spaces differing by 2 in $N_{\text {ex }}$.

If $\mathrm{Sp}(3, R)$ dynamical symmetry holds for the nucleus, states will be organized into $\mathrm{Sp}(3, R)$ irreps. Here we have the subgroup chain $\mathrm{Sp}(3, R) \supset \mathrm{U}(3) \sim \mathrm{U}(1) \times \mathrm{SU}(3) \supset$ $\mathrm{SO}(3)$. The U(3) group here is simply obtained by combining Elliott's $\mathrm{SU}(3)$ with the trivial $\mathrm{U}(1)$ group of the harmonic oscillator Hamiltonian $\hat{H}_{0}$. The set of states in a $\mathrm{U}(3)$ irrep (i.e., the invariant subspace) is the same as for an Elliott $\mathrm{SU}(3)$ irrep, but the new operator $\hat{H}_{0}$ provides a further label - the number of oscillator quanta or, equivalently, $N_{\mathrm{ex}}$ - beyond the usual SU(3) labels $(\lambda, \mu)$. The number of oscillator quanta is relevant in the context of $\operatorname{Sp}(3, R)$ dynamical symmetry, as an $\operatorname{Sp}(3, R)$ irrep spans many, in fact, infinitely many, $N_{\text {ex }} \hbar \omega$ spaces. One builds an $\operatorname{Sp}(3, R)$ irrep $\sigma=N_{\sigma, \text { ex }}\left(\lambda_{\sigma}, \mu_{\sigma}\right)$ recursively, starting from one Elliot $\mathrm{SU}(3)$ irrep $\left(\lambda_{\sigma}, \mu_{\sigma}\right)$, at some lowest number $N_{\sigma, \mathrm{ex}}$ of oscillator excitations. Then by repeatedly laddering with the $\mathrm{Sp}(3, R)$ raising operator $\hat{A}$ one obtains an infinite tower of $\mathrm{U}(3)$ irreps with different $N_{\mathrm{ex}}=N_{\sigma, \mathrm{ex}}, N_{\sigma, \mathrm{ex}}+2, N_{\sigma, \mathrm{ex}}+4$, and so on.

When one moves beyond simple algebraic models, such as Elliott's for SU(3) or analogous algebraic models for $\operatorname{Sp}(3, R)[58]$, the interaction may break the dynamical symmetry and mix the wave function across irreps. While one might expect the mixing to be state dependent, surprisingly often one finds similar patterns of mixing across multiple states $[36,59]$. This is quasi-dynamical symmetry [60-62].

\subsection{But which symmetry?}

Now we come to the main motivation for this work. The Elliott SU(3) model has a long and successful history of describing rotational bands. Yet it has a natural limitation: $\mathrm{SU}(3)$ irreps are constrained to configurations involving the valence shell, or, more generally, they cannot combine configurations from different shell model $N_{\text {ex }} \hbar \omega$ spaces. If one expects low-lying states to be dominated by valence subspace configurations, and the valence subspace has a maximum angular momentum, then the rotational band should terminate at that maximum angular momentum.

A long-standing question has been of extended bands, that is, bands that contain members outside the valence subspace. An early phenomenological study of ${ }^{8} \mathrm{Be}$ and ${ }^{20} \mathrm{Ne}$ based upon particle-hole excitations (and not in an algebraic picture) found only weak $E 2$ transitions and thus no extended band [17]. Later phenomenological [but using an $\operatorname{Sp}(3, R)$ framework] calculations of ${ }^{20} \mathrm{Ne}[18,19]$, as well as ab initio NCSM calculations of beryllium isotopes [20], found evidence for extended rotational bands: strong transitions from states outside the valence subspace, and in particular beyond the maximally allowed valence-subspace angular momentum, to ground-state band members.

While this violates the Elliott $\mathrm{SU}(3)$ picture, note that the physical electric quadrupole (E2) transition operator [43], proportional to $\sum_{i} e_{i} r_{i}^{2} Y_{2 m}\left(\hat{r}_{i}\right)$ where $e_{i}$ is the charge of the $i$ nucleon, connects configurations of different $N_{A}$, while the Elliott quadrupole operator (3), cannot. Because the isoscalar component of the $E 2$ transition operator is encompassed in $\operatorname{Sp}(3, R)$, we choose to carry out a sideby-side comparison of $\mathrm{SU}(3)$ and $\mathrm{Sp}(3, R)$ symmetry for such bands. Specifically, we take NCSM calculations of several beryllium isotopes, as well as of ${ }^{20} \mathrm{Ne}$, and, using previously developed techniques, decompose the wave functions into the irreps of Elliott's $\mathrm{SU}(3)$ and of $\mathrm{Sp}(3, R)$. Note that the NCSM calculations make no assumptions about the underlying group structure. 


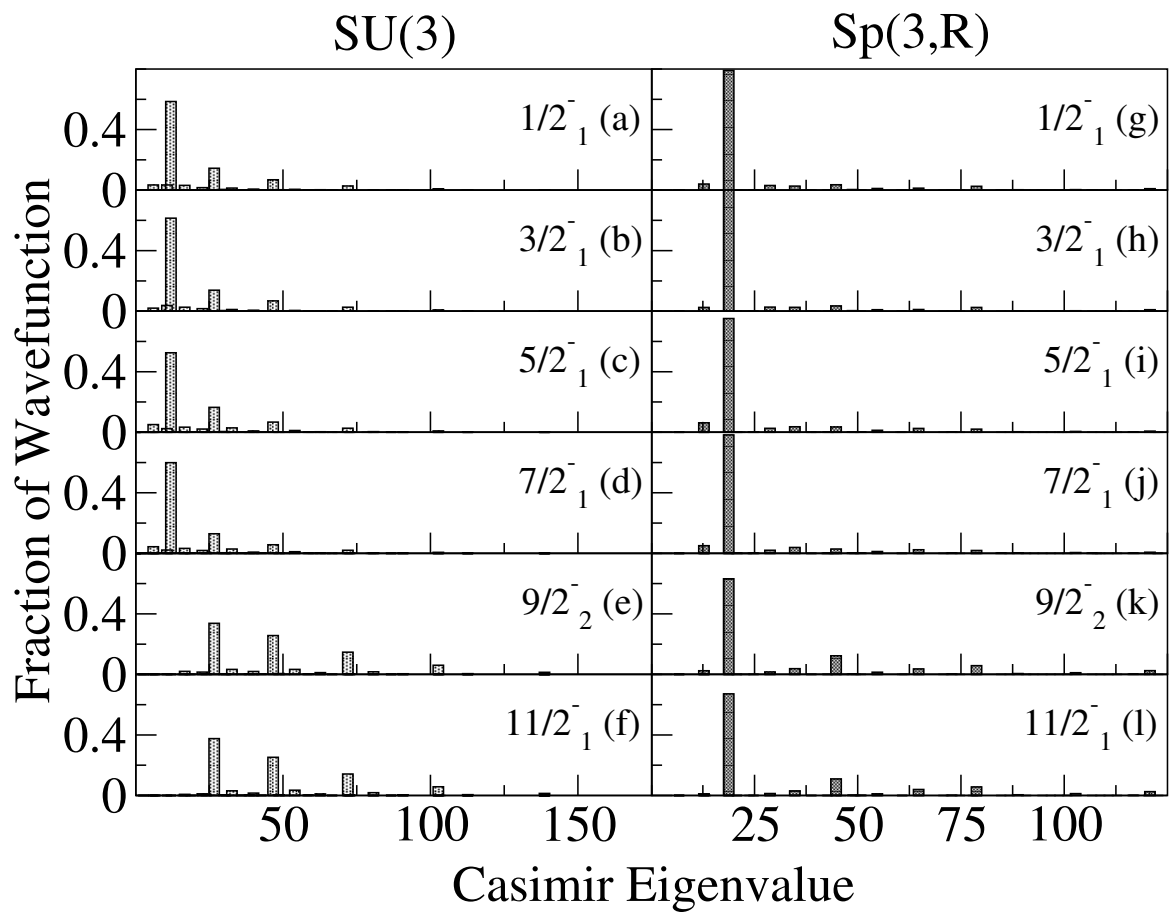

Figure 2: Algebraic decompositions of the ground-state band of ${ }^{7}$ Be shown in Fig. 1. Left-hand panels (a)-(f): decomposition by the quadratic Casimir of SU(3). Righthand panels (g)-(l): decomposition by the quadratic Casimir of $\operatorname{Sp}(3, R)$. The maximal angular momentum within the lowest harmonic oscillator configuration, the so-called valence subspace, is $J=7 / 2$.

One can adapt the Lanczos algorithm to decompose any wave function into its contributions from subspaces defined by the eigenvalues of any Hermitian operator [63]. Thus, to find $\mathrm{SU}(3)$ or $\mathrm{Sp}(3, R)$ decompositions of an NCSM wave functions obtained in an ordinary $M$-scheme basis, we make use of the Casimir operator for the given group. Lanczos-enabled decompositions have been used before to investigate quasidynamical symmetry [36-38].

A Casimir operator $\hat{C}$ is built from the generators of a given group, constructed so as to commute with all the generators (equivalently, it is invariant under the symmetry operations of the group). It therefore acts as a constant multiple of the identity operator within any irrep, and has an eigenvalue which depends only upon the quantum numbers labeling the irrep: $\hat{C}|\alpha \Gamma i\rangle=g(\Gamma)|\alpha \Gamma i\rangle$. For the familiar case of $\mathrm{SU}(2)$, the quadratic Casimir operator is $\hat{C}_{2}[\mathrm{SU}(2)]=\hat{\mathbf{J}} \cdot \hat{\mathbf{J}}$, with eigenvalue $g(J)=J(J+1)$. The quadratic Casimir operator of $\mathrm{SU}(3)$ is, to within an arbitrary choice of normalization $[5,15,64]$,

$$
\hat{C}_{2}[\mathrm{SU}(3)]=\frac{1}{6}[\hat{\mathcal{Q}} \cdot \hat{\mathcal{Q}}+3 \hat{\mathbf{L}} \cdot \hat{\mathbf{L}}]
$$

which has eigenvalue

$$
g(\lambda, \mu)=\frac{2}{3}\left(\lambda^{2}+\lambda \mu+\mu^{2}+3 \lambda+3 \mu\right) .
$$


The quadratic $\operatorname{Sp}(3, R)$ Casimir operator for $\operatorname{Sp}(3, R)$ is [64]

$$
\hat{C}_{2}[\mathrm{Sp}(3, R)]=\hat{C}_{2}[\mathrm{SU}(3)]-2 \hat{A}_{2} \cdot \hat{B}_{2}-2 \hat{A}_{0} \cdot \hat{B}_{0}+\frac{1}{3} \hat{H}_{0}^{2}-4 \hat{H}_{0},
$$

with eigenvalue

$$
g(\sigma)=\frac{2}{3}\left(\lambda_{\sigma}^{2}+\mu_{\sigma}^{2}+\lambda_{\sigma} \mu_{\sigma}+3 \lambda_{\sigma}+3 \mu_{\sigma}\right)+\frac{N_{\sigma}^{2}}{3}-4 N_{\sigma} .
$$

The basic idea, then, is that the full nuclear many-body space can be broken into subspaces identified with an eigenvalue of the Casimir operator. This normally corresponds to selecting a particular value for the irrep label $\Gamma$, though there can be ambiguities: e.g., for $\mathrm{SU}(3)$, irreps with labels $(\lambda, \mu)$ or $(\mu, \lambda)$ have the same eigenvalue for the quadratic Casimir operator (5). [One can, in principle, separate out contributions from these irreps by using higher-order Casimir operators, such as the cubic Casimir for $\mathrm{SU}(3)$, which break the degeneracy, but this involves going to three-body or higher-body operators in the shell model calculation.]

One can adapt the Lanczos algorithm to compute the fraction $\mathcal{F}(\Gamma)$ of a given wave function, in any such subspace $\Gamma$ [36-38]. This is equivalent to projecting the state $|\Psi\rangle$ onto a full group theoretical basis:

$$
\mathcal{F}(\Gamma)=\sum_{\alpha, i}|\langle\alpha \Gamma i \mid \Psi\rangle|^{2} .
$$

For instance, for $\mathrm{SU}(3)$, in finding the fraction from $\Gamma \rightarrow(\lambda, \mu)$, we implicitly aggregate all contributions from the many ways this $(\lambda, \mu)$ can be obtained as the result of different shell model configurations and intermediate couplings, as well as the contributions from different states $i$ within each irrep. Luckily, the Lanczos method efficiently accomplishes this without any need to explicitly construct a group theoretical basis.

In this paper we provide decompositions by plotting $\mathcal{F}(\Gamma)$, the fraction of wavefunction in the subspace, versus $g(\Gamma)$. An example is in Fig. 2. As we follow members of the ground state band, defined by strong $E 2$ transitions, to higher angular momentum, we find, as expected, that the Elliott SU(3) decompositions change abruptly as one exits the valence subspace, that is, as the band extends to angular momenta not contained in the valence subspace. Decompositions by the $\operatorname{Sp}(3, R)$ quadratic Casimir, on the other hand, are nearly identical across the valence subspace boundary, providing a more consistent representation of the members of a band than those by the Elliott $\mathrm{SU}(3)$ Casimir. This is the overarching theme of this paper.

Furthermore, as can be seen in Fig. 1, the $E 2$ transitions connect not only the yrast band, but also extends to an upper band consisting of excited states connected by strong $B(E 2)$ values. In Fig. 3 we decompose these states: these states share the same $\mathrm{Sp}(3, R)$ decomposition, but a very different (albeit consistent) $\mathrm{SU}(3)$ decomposition from the ground-state band $[32,34]$. We show this pattern persists not only in several beryllium isotopes, but also in ${ }^{20} \mathrm{Ne}$. Because the two bands unite at high angular momentum and share the same symplectic decomposition, we argue they are really a single unified feature rather than independent bands.

\section{Results}

In this section we discuss in some detail our results, working through each of the studied nuclides one by one. 


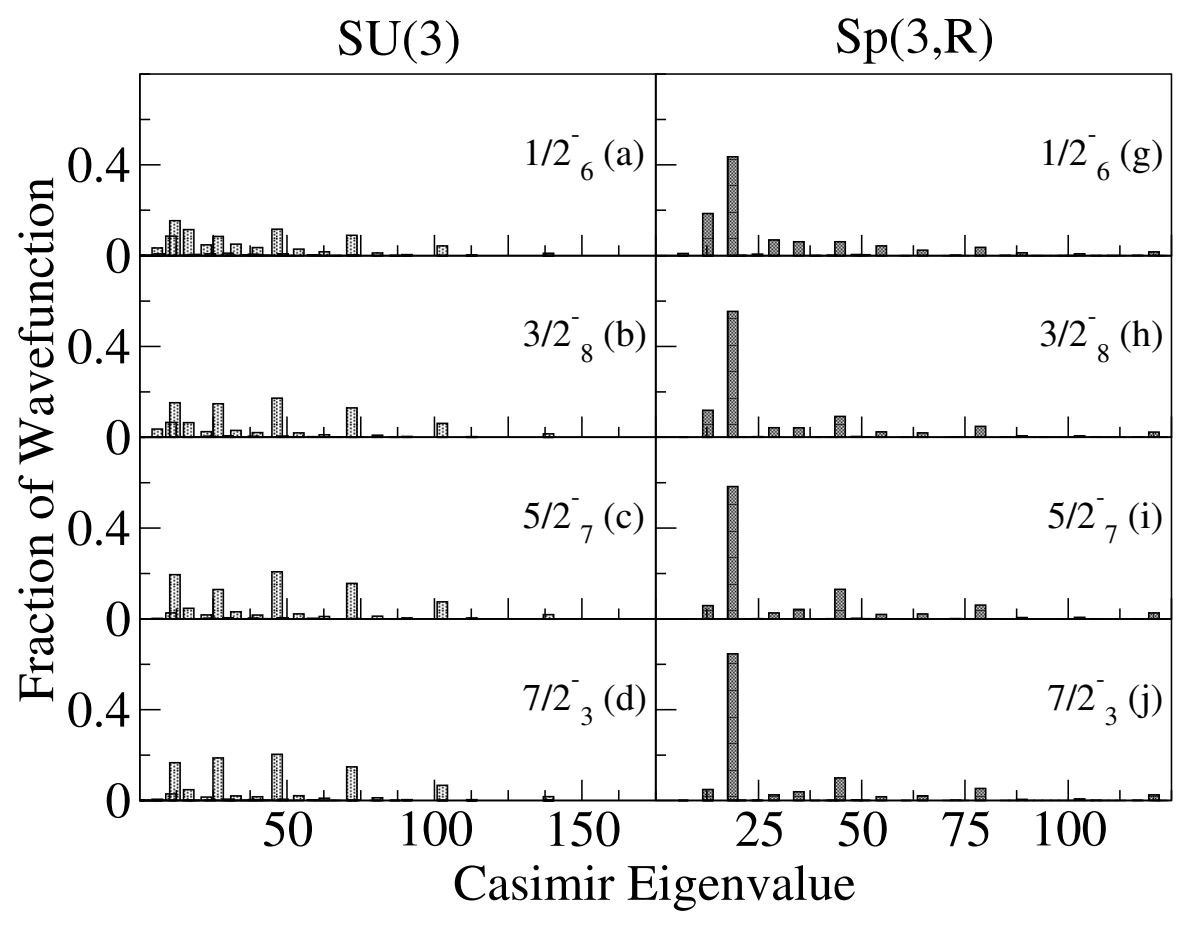

Figure 3: Decompositions of members of the upper rotational band of ${ }^{7}$ Be shown in Fig. 1. Left-hand panels, (a)-(f): Decomposition by the quadratic Casimir of $\mathrm{SU}(3)$. Right-hand panels,(g)-(l): decomposition by the quadratic Casimir of $\mathrm{Sp}(3, R)$. Although not shown, these states are all dominated by $N_{\mathrm{ex}}=2(2 \hbar \omega)$ configurations.

\section{1. ${ }^{7} \mathrm{Be}$}

Previous works $[20,34,39,40,42]$ have made detailed investigations into ${ }^{7} \mathrm{Be}$ as a rotational nucleus in $a b$ initio calculations, looking carefully at ratios of $B(E 2)$ values, $M 1$ transitions, decomposition of the wavefunction into total orbital angular momentum $L$ and total spin $S$, and so on. Those calculations were made using the JISP16 [65], NNLO opt [66], and Daejeon16 [67] interactions. While we follow in their footsteps, we use an interaction derived from an N3LO chiral effective field theory [35], and we simply identify band structure through strong $B(E 2)$ values.

Fig. 1 shows both our calculated excitation spectrum (with known experimental levels [68] marked for comparison) and, through strong $B(E 2) \mathrm{s}$, clear band structure. The ground-state band of ${ }^{7} \mathrm{Be}$ has the Coriolis staggering typical of $K=1 / 2$ bands, where $K$ is the $z$-projection of angular momentum in the intrinsic frame [2], with an inverted angular momentum sequence $\left(3 / 2_{1}^{-}, 1 / 2_{1}^{-}, 7 / 2_{1}^{-}, 5 / 2_{1}^{-}\right)$. Later on we will look at another $K=1 / 2$ band in the unnatural parity space of ${ }^{9} \mathrm{Be}$.

We plot the downward $B(E 2)$ strengths as solid lines, with the width and shading approximately proportional to the log strength, for $B(E 2)$ values above $1.5 e^{2} \mathrm{fm}^{4}$. It is also important to note in Fig. 1 many downward transitions drawn between states are not part of the identified bands. This is done to illustrate how we use $E 2$ transition network plots as a qualitative tool to effectively locate theoretical rotational structure amongst many other transitions. However, for nuclei in the following sections, we omit 


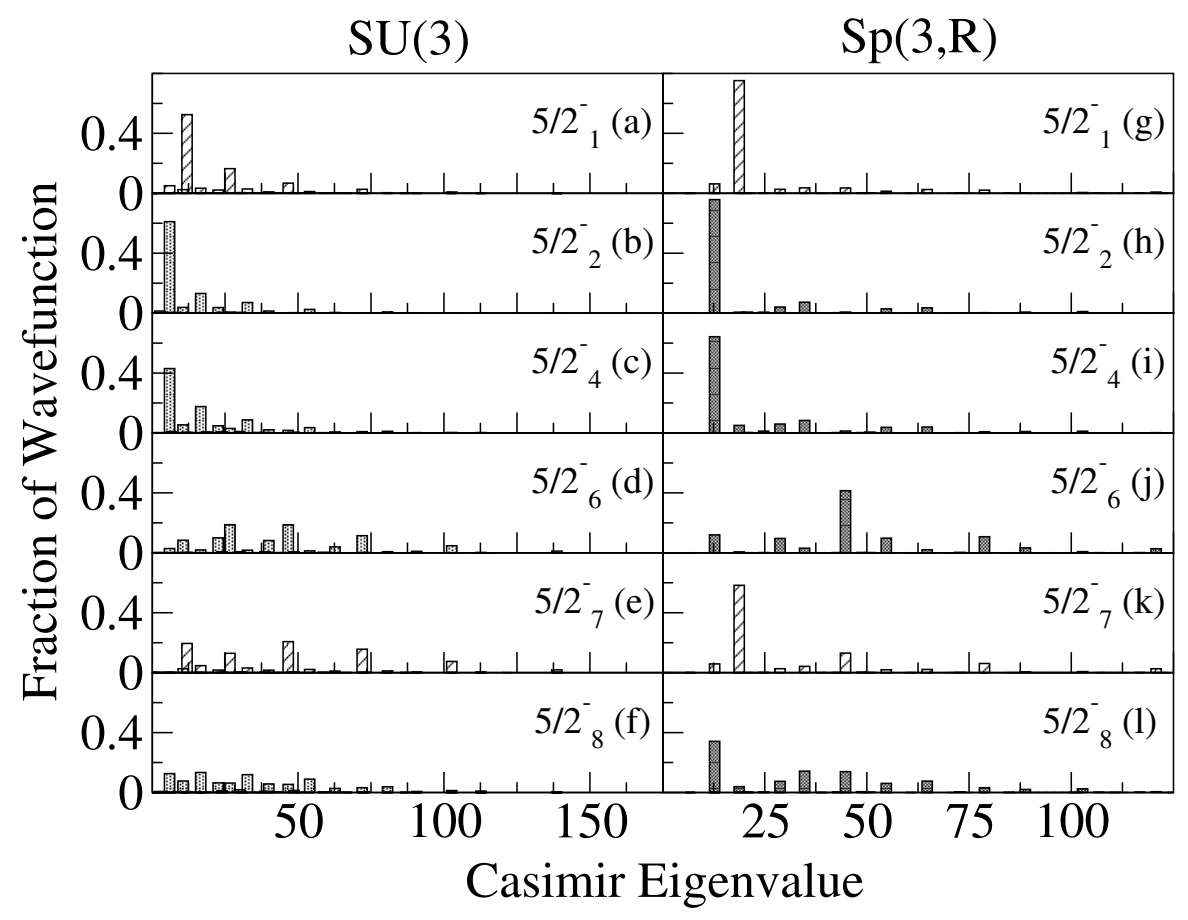

Figure 4: Decompositions of $5 / 2^{-}$states of ${ }^{7} \mathrm{Be}$. Left-hand panels (a)-(f): decomposition by the quadratic Casimir of SU(3). Right-hand panels (g)-(l): decomposition by the quadratic Casimir of $\operatorname{Sp}(3, R)$. Decompositions duplicated for purposes of comparison from Fig. 1 , that is, the $5 / 2_{1}^{-}$and $5 / 2_{7}^{-}$states, are marked with stripes.

such $E 2$ transitions not involving states we have identified as members of rotational bands.

As discussed in Sec. 2.1, a key concept is the 'valence subspace' defined by the lowest harmonic oscillator configurations, that is, $N_{\mathrm{ex}}=0$ or $0 \hbar \omega$ configurations. For ${ }^{7} \mathrm{Be}$ and the other beryllium isotopes we consider, the valence subspace consists of an inert ${ }^{4} \mathrm{He}$ core and the remaining nucleons restricted to the $0 p$ shell. In particular we are interested in the maximal angular momentum in the valence subspace, which is $J=7 / 2$ for ${ }^{7} \mathrm{Be}$. Hence any state with $J>7 / 2$ must be outside the valence subspace. In Fig. 1, and subsequent similar figures, we denote the maximal $J$ in the valence space by a vertical dashed line.

Given the limit in angular momentum in the valence subspace, it is unsurprising that the $\mathrm{SU}(3)$ decompositions shown in Fig. 2 (a)-(f) express an abrupt change in dominant irreps as one goes from the $7 / 2_{1}^{-}$state to the $9 / 2_{2}^{-}$state. On the basis of Elliott's SU(3) model, then, one would naively expect that the ground-state band should terminate at $J=7 / 2$. But the $B(E 2)$ values show strong transition strengths to both the $9 / 2_{2}^{-}$and $11 / 2_{1}^{-}$states, and hence by that traditional criteria these states are candidates for belonging to the same band. Yet $9 / 2_{2}^{-}$and $11 / 2_{1}^{-}$states of the lowest band are, of necessity, outside the valence subspace, and are dominated by $N_{\text {ex }}=2$ configurations [42]. We remind the reader that within a single $\operatorname{Sp}(3, R)$ irrep 
one can have states belonging to different $\mathrm{SU}(3)$ irreps, involving different numbers $N_{\text {ex }}$ of oscillator excitations.

It is very satisfactory, then, to see this analysis borne out when decomposing in $\mathrm{Sp}(3, R)$, in the right-hand panels Fig. 2(g)-(l). All of the states in the band show nearly identical decompositions, and indeed are close to a pure dynamical symmetry. This provides evidence $\operatorname{Sp}(3, R)$ is a more unifying symmetry for rotational bands than Elliott's $\mathrm{SU}(3)$. Of course, this was the original motivation for $\operatorname{Sp}(3, R)$ [21]; as in references [33,34], we see this relationship emerge without any assumptions, starting from an independent $a b$ initio nuclear interaction and relying upon $B(E 2)$ values to identify bands.

The ground-state band does not exist in isolation: there is an upper band [34], connected by strong $B(E 2)$ s, including the $1 / 2_{6}^{-}, 3 / 2_{8}^{-}, 5 / 2_{7}^{-}$and $7 / 2_{3}^{-}$as shown in Fig. 1. (This is for our $N_{\max }=10$ calculations. Because of the high density of excited states, the exact position of these members of the excited band will depend sensitively upon the details of the calculation, not only the model space and singleparticle basis frequency, but also upon the choice of interaction. Specifically, we do not claim, for example, that the physical $3 / 2_{8}^{-}$state is a member of the band, only that there exists a highly excited $3 / 2^{-}$state which is a member of the band. Our broader narrative, however, that is the existence of a strongly connected upper band, is robust and insensitive to these details.) These two 'bands', as traced out by E2 transitions, appear to share members at high angular momentum, here $J=11 / 2$, a pattern we will see repeated in other nuclei.

We decompose the states identified as members of the upper band in Fig. 3. The $\mathrm{SU}(3)$ decompositions for the $1 / 2_{6}^{-}, 3 / 2_{8}^{-}, 5 / 2_{7}^{-}$, and $7 / 2_{3}^{-}$states, Fig. $3(\mathrm{a})-(\mathrm{d})$, while highly fragmented, are nonetheless similar to each other, but very different from the $\mathrm{SU}(3)$ decompositions of the ground-state band. The decompositions by $\operatorname{Sp}(3, R)$, Fig. 3(g)-(l), tell a different story. A distinct dominant $\operatorname{Sp}(3, R)$ irrep is shared across all the states, and despite some fragmentation in the $1 / 2_{6}^{-}$and $3 / 2_{8}^{-}$in Fig. $3(\mathrm{~g})$, (h), which we attributed to mixing due to the high local density of states with similar $J^{\pi}$, the upper band decompositions directly resemble the ground-state band in Fig 2 .

To show that $\operatorname{Sp}(3, R)$ differentiates band members from states outside the band, we decompose several $5 / 2^{-}$states in Fig. 4 . The $5 / 2_{1}^{-}$and $5 / 2_{7}^{-}$states have already been discussed as members of the ground-state band and upper band, respectively. Recall that the states in these bands have similar $\operatorname{Sp}(3, R)$ decompositions [as seen in Fig. $4(\mathrm{~g}),(\mathrm{k})$ ] even though the $\mathrm{SU}(3)$ decompositions have little in common [as seen in Fig. 4 (a), (e)]. The other $5 / 2^{-}$states, not members of either band, have very different $\operatorname{Sp}(3, R)$ decompositions. We have carried out other similar studies, i.e., decomposed multiple excited states of the same angular momentum and parity with the same result: states outside the band have dramatically different $\operatorname{Sp}(3, R)$ decompositions.

Our calculations also naturally determine the fraction of the wave function for each $N_{\mathrm{ex}}$ (or $\left.N_{\mathrm{ex}} \hbar \omega\right)$. As prior work [42] discusses the distribution in $N_{\text {ex }}$ in more detail, we only touch upon the highlights. As we increase $N_{\max }$, the fraction of the wave function in the valence subspace naturally decreases. For example, at $N_{\max }=2$ the yrast band states have $\sim 85 \%$ of their probability coming from $N_{\text {ex }}=0$ configurations, while at $N_{\max }=6$ this contribution falls to $\sim 71 \%$. By $N_{\max }=10$, where the distribution appears to stabilize, yrast states with $J \leq 7 / 2$ still had $\sim 60 \%$ of their wave function within the valence subspace.

By contrast, the highly excited $5 / 2_{7}^{-}$state has roughly $20 \%$ of its wavefunction 
in each of the $N_{\mathrm{ex}}=0$ and $N_{\mathrm{ex}}=2$ spaces and $26 \%$ in the $N_{\mathrm{ex}}=4$ space. Correspondingly, the $5 / 2_{7}^{-}$has a very different $\mathrm{SU}(3)$ decomposition from the yrast $5 / 2_{1}^{-}$which has the largest fraction of its wave function in the valence subspace. Yet, as we have already noted, the $5 / 2_{1}^{-}$and $5 / 2_{7}^{-}$share nearly identical $\operatorname{Sp}(3, R)$ decompositions.

This trend continues: the $5 / 2_{2}^{-}$and $5 / 2_{4}^{-}$states, like the $5 / 2_{1}^{-}$state, have wave functions with a largest fraction in the valence subspace, and have similar decompositions in $\mathrm{SU}(3)$, Fig. 4 (b), (c) and $\mathrm{Sp}(3, R)$, Fig. 4 (h), (i). Conversely, $5 / 2_{6}^{-}$is the first $5 / 2^{-}$state to be primarily outside the valence subspace, with only a few percent contribution from $N_{\text {ex }}=0$, and $\sim 30 \%$ from $N_{\text {ex }}=2$ and $N_{\text {ex }}=4$ configurations each. Correspondingly one can see the decompositions in both $\mathrm{SU}(3)$, in Fig. 4 (d), and $\mathrm{Sp}(3, R)$, in Fig. $4(\mathrm{j})$, are very different from the lower-lying $5 / 2^{-}$ states.

The above calculation was carried out at $N_{\max }=10$. As $N_{\max }$ increases, although the ground-state energy (or binding energy) evolves significantly, excitation energies of the states which lie primarily in the valence subspace converge very quickly (see, e.g., figure 6 of reference [42] for illustration). The excitation energies of the states involving primarily contributions from higher $N_{\text {ex }}$ converge more slowly (figure 17 of reference [42]). The decompositions, however, are generally robust with respect to $N_{\max }$ and, to a lesser extent, the basis oscillator frequency, as detailed in the Appendix (figures A1 and A2).

We consider three more nuclides to demonstrate these patterns are not a singular phenomenon.

\section{2. ${ }^{8} \mathrm{Be}$}

The strongly-connected positive parity $K=0$ [40] yrast band for ${ }^{8} \mathrm{Be}$, seen in Fig. 5, is not surprising, given that one can think of ${ }^{8} \mathrm{Be}$ as two $\alpha$ particles in a dumbbell configuration [70]. Nonetheless, the story here echoes that of ${ }^{7} \mathrm{Be}$.

The maximum angular momentum in the valence subspace is $J=4$, but our calculated $B(E 2)$ s clearly demarcate a band extending out of the valence subspace to the $6_{1}^{+}$and $8_{2}^{+}$states. Investigation of a possible extension of the ground-state band in ${ }^{8} \mathrm{Be}$ to extra-valence states go back at least fifty years. While a two-particle, two-hole calculation of extra-valence states found only weak $B(E 2)$ values, suggesting there was not an extended band [17], other calculations supported an extended band. These include projected Hartree-Fock calculations [71] and configuration-interaction calculations investigating the mixing of $\mathrm{SU}(3)$ [72]. In particular early multi- $N_{\mathrm{ex}}$ calculations [73] found, like us, lower and upper bands; this led to the use of $\operatorname{Sp}(2, R)$ $[74,75]$ and prefigured later use of $\operatorname{Sp}(3, R)$. Finally, more recent investigations in NCSM frameworks likewise found the $6_{1}^{+}$and $8_{2}^{+}$states to be members of the ground-state band [20,33], and identified an upper band having very similar $\operatorname{Sp}(3, R)$ decompositions to the ground-state band [33].

Fig. 6(a)-(j) (left-hand plots) clearly shows that decompositions with $\operatorname{Sp}(3, R)$ provide a more unifying picture than with $\mathrm{SU}(3)$. Decompositions with Elliott's $\mathrm{SU}(3)$ are nearly identical for the yrast states within the valence subspace, but change dramatically for $J>4$ states outside the valence subspace. There is some evolution of the $\operatorname{Sp}(3, R)$ decomposition also for $J>4$ ground-state band members, but much less dramatic. Note that while we went up to $N_{\max }=10$, the rate of convergence with increasing $N_{\max }$ for the energies of the extra-valence $6_{1}^{+}$, and $8_{2}^{+}$states is significantly 


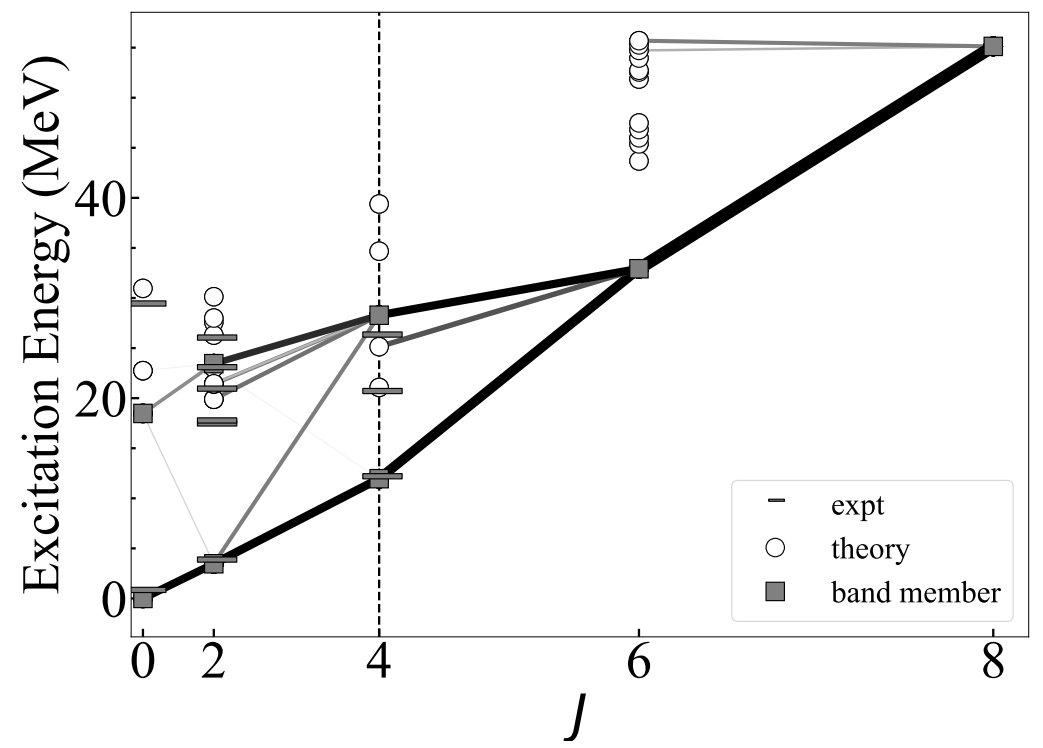

Figure 5: Excitation spectrum of the natural positive parity states of ${ }^{8} \mathrm{Be}$, computed at $N_{\max }=10$ with a single-particle harmonic oscillator basis frequency $\hbar \omega=20 \mathrm{MeV}$, using a two-body chiral force at N3LO. Experimental energies [69] are presented as shaded bars for comparison (see text). Shaded squares indicate states decomposed into group irreps in Fig. 6. Downward (high to low $J$ ) E2 transitions connecting to band members are indicated with solid lines. Line thickness and shading is proportional to $B(E 2)$ strength. Only strengths above $0.1 e^{2} \mathrm{fm}^{4}$ are shown. The vertical dashed line denotes the maximal angular momentum within the lowest harmonic oscillator configuration.

slower than for states with $J \leq 4$, much as we found with other nuclides.

Strong $E 2$ transitions clearly link the upper band, $0_{2}^{+}, 2_{8}^{+}, 4_{5}^{+}$at $N_{\max }=10$, to the ground-state band. Fig. 6 (aa)-(ff) (right-hand plots) presents decompositions of this upper band. The $\operatorname{Sp}(3, R)$ decompositions of the upper band members in Fig. 6(dd)-(ff) are very similar to those of the ground-state band, albeit with some fragmentation. The fragmentation is especially noticeable for the $2_{8}^{+}$state, which could be due to mixing with nearby states, while the SU(3) decompositions in Fig. 6(aa)(cc) are highly fragmented. We note the valence yrast $\left(0_{1}^{+}, 2_{1}^{+}\right.$and $\left.4_{1}^{+}\right)$wave functions have $N_{\mathrm{ex}}=0$ contributions of roughly $60 \%$, similar to that of ${ }^{7} \mathrm{Be}$, while members of the upper band with $J \leq 4$ had a smaller fraction of their wave functions in the $N_{\text {ex }}=0$ space, between $20-45 \%$. This follows prior work using a symplectic $N_{\max }=4$ basis that found the upper band states and the $6_{1}^{+}$and $8_{2}^{+}$states have similar $U(3)$ decompositions [33].

Of course, ${ }^{8} \mathrm{Be}$ is unbound, so that neither the $6^{+}$nor $8^{+}$states have been experimentally detected, and the continuum likely plays a role we leave unexplored. Instead, we emphasize here the similarities to ${ }^{7} \mathrm{Be}$. 


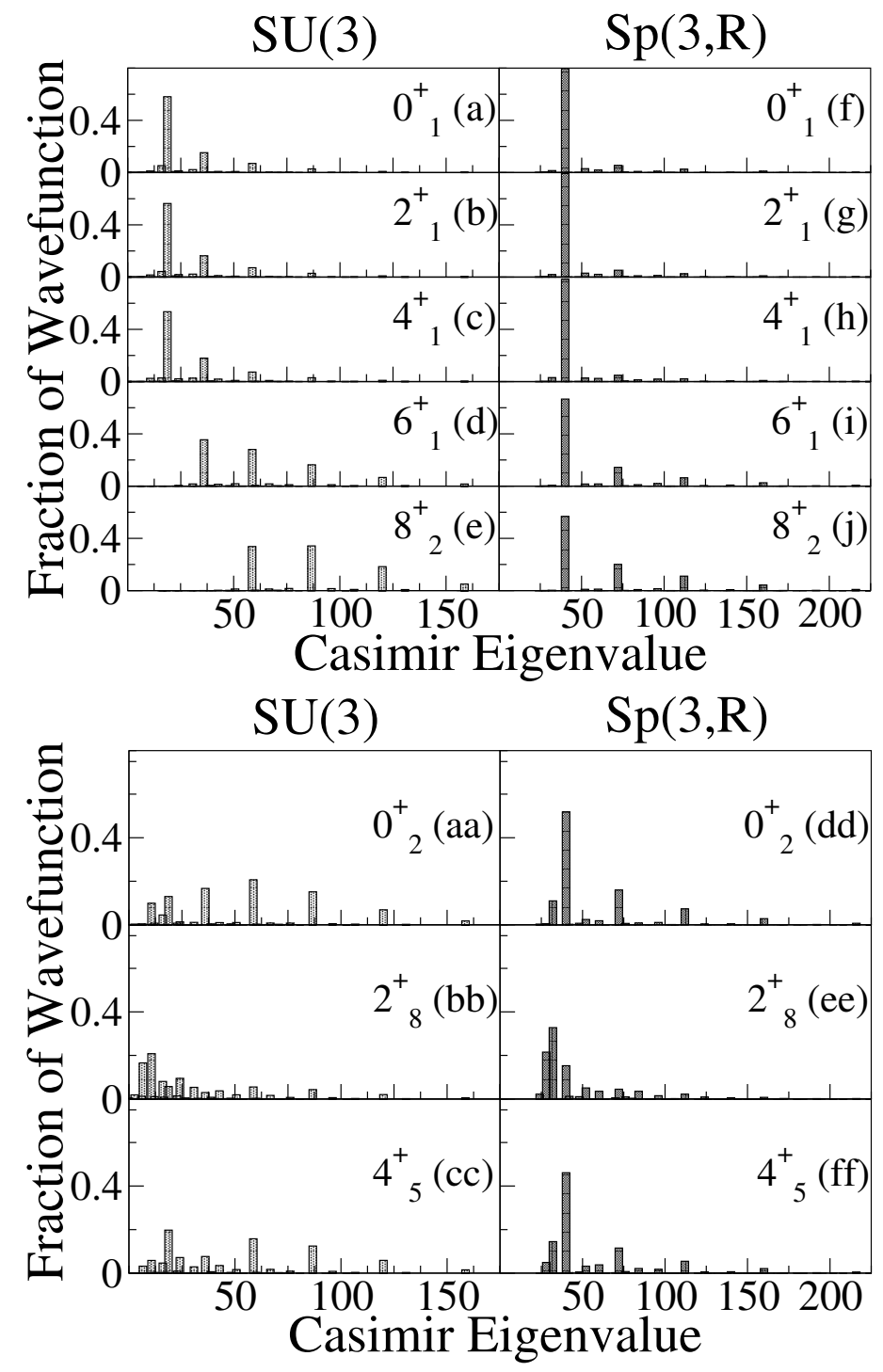

Figure 6: Decompositions by the quadratic Casimir of $\mathrm{SU}(3)$ and $\mathrm{Sp}(3, R)$ of ${ }^{8} \mathrm{Be}$ band members as calculated in Fig. 5. Ground-state decompositions are shown in panels (a)-(j). Excited band decompositions are shown in panels (aa)-(ff).

\section{3. ${ }^{9} \mathrm{Be}$}

An early application of $\mathrm{SU}(3)$ to light nuclei was to ${ }^{9} \mathrm{Be}[76]$. While the negative parity ground-state band is $K=3 / 2$ [77], the first excited state is the bandhead of an unnatural positive parity $K=1 / 2$ band, as explained by projected Hartree-Fock calculations [78]. Given the $2 \alpha$ structure of ${ }^{8} \mathrm{Be}$, it is reasonable to picture ${ }^{9} \mathrm{Be}$ as two $\alpha$ particles plus an extra orbiting neutron [79]. Although the first NCSM calculations of ${ }^{9} \mathrm{Be}[80]$ did not focus on the rotational motion, three rotational bands have been documented in the calculated spectra of ${ }^{9} \mathrm{Be}[20,39,40,42]$. Two of these bands are in 


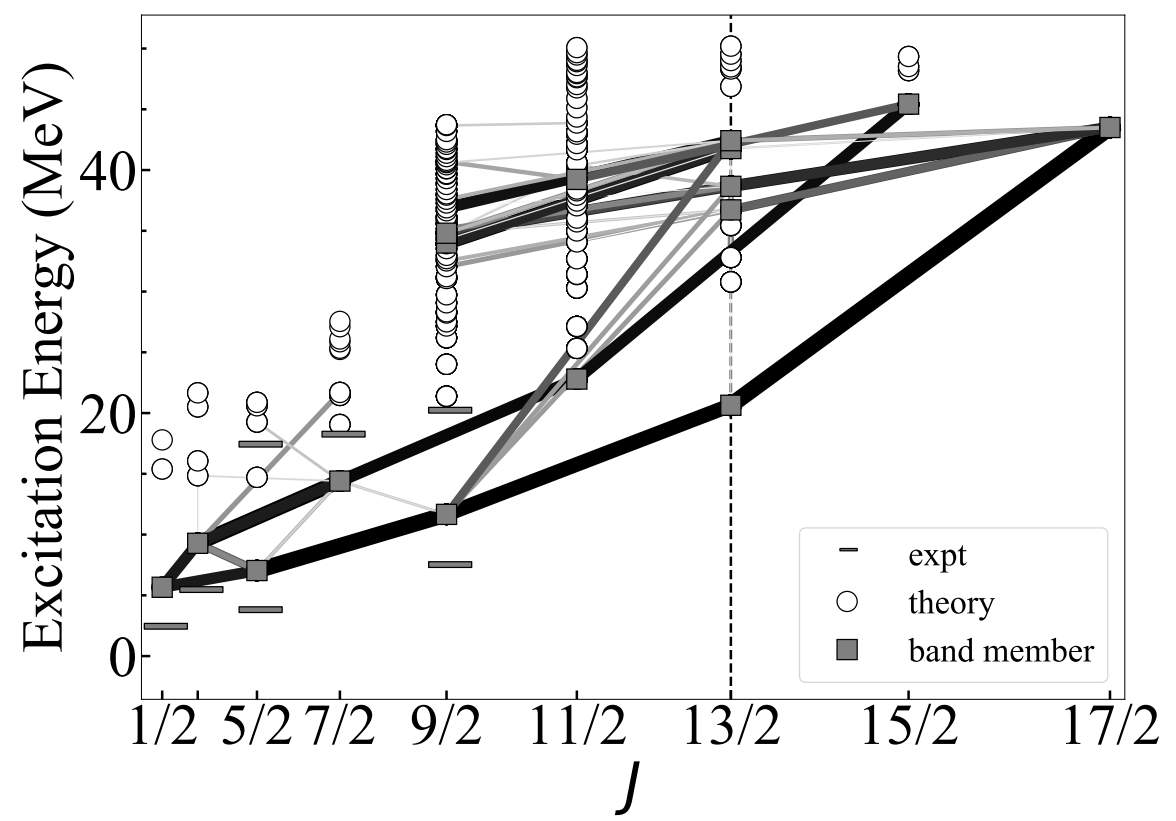

Figure 7: Excitation spectrum of the unnatural, positive parity states of ${ }^{9} \mathrm{Be}$, computed at $N_{\max }=9$ with a single-particle harmonic oscillator basis frequency $\hbar \omega=20 \mathrm{MeV}$, using a two-body chiral force at N3LO. (The natural parity states are not shown.) Excitation energies are calculated with respect to the natural (negative) parity ground state. Experimental energies are presented as shaded bars for comparison (see text). Shaded squares indicate states we decompose into group irreps in Fig. 8 and 9. $J$-changing $E 2$ transitions connecting to band members are indicated with solid lines. Line thickness and shading is proportional to $B(E 2)$ strength. Only strengths above $2.0 e^{2} \mathrm{fm}^{4}$ are shown. The vertical dashed line denotes the maximum angular momentum in the $N_{\mathrm{ex}}=1$ space.

the natural (negative) parity space, and the third is within the unnatural (positive) parity space. To emphasize the generality of our findings, we study here the unnatural parity states shown in Fig. 7. Once again we see what appear to be upper and lower band-like structures meeting at high angular momentum $J$. The lower $K=1 / 2$ band is well isolated from higher-lying states and shows the characteristic Coriolis staggering.

We carry out our positive parity calculations in an $N_{\max }=9$ space. However, the natural parity $3 / 2^{-}$ground state must be calculated in an even $N_{\max }$ space, so, to obtain the zero point for excitation energies in Fig. 7, we average the groundstate energies obtained in $N_{\max }=8$ and 10 calculations. The calculated excitation energies for positive parity states at $N_{\max }=9$ are still significantly higher than the experimental energies from [69] (also shown in Fig. 7), much as in other calculations [80]. However, these energies are expected to continue to converge downward towards the experimental energies with increasing $N_{\max }[42,81]$.

Although all the positive parity states are outside the natural-parity valence subspace, the $N_{\mathrm{ex}}=1$ space plays the analogous role to the valence subspace 


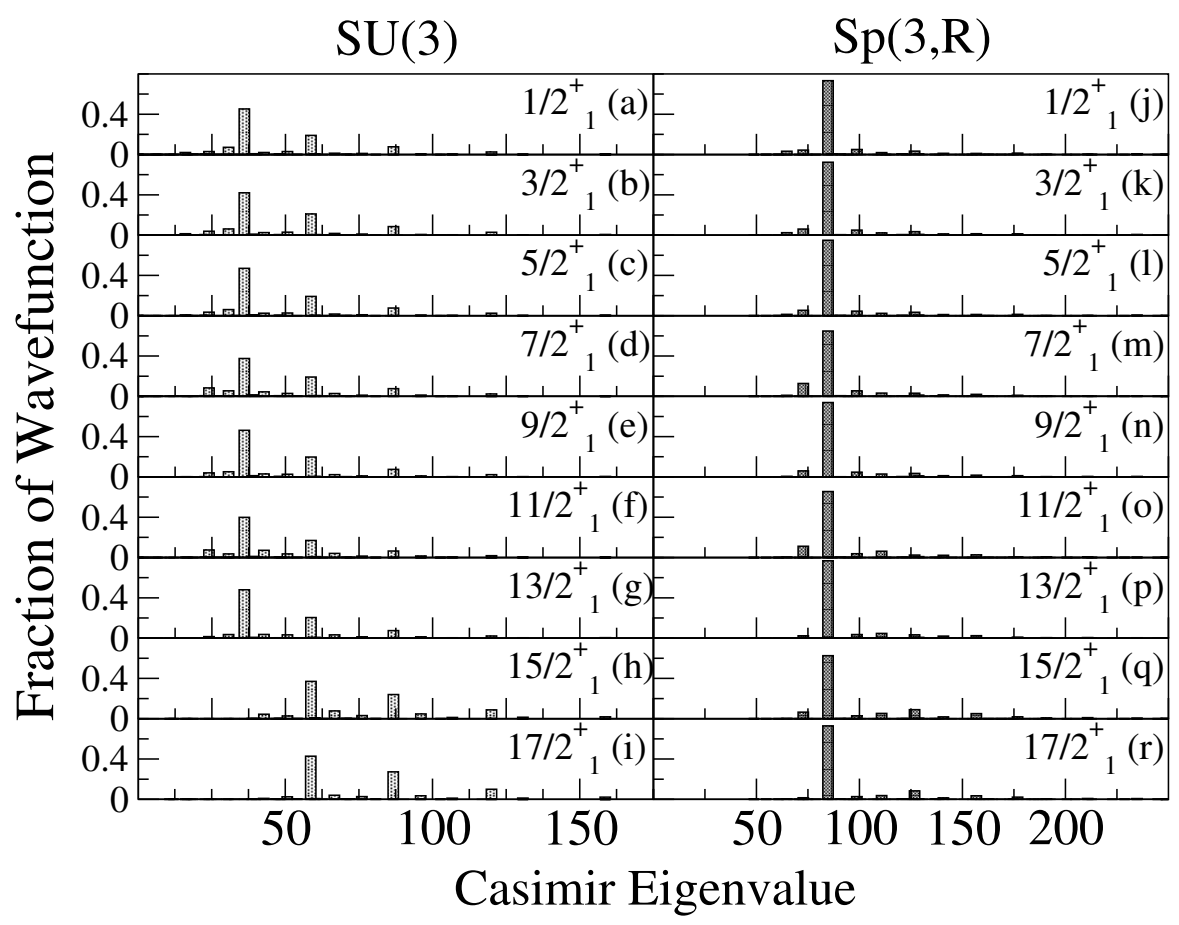

Figure 8: Decompositions of the members of the lower rotational band of ${ }^{9} \mathrm{Be}$ shown in Fig. 7. Left-hand panels, (a)-(i): Decomposition by the quadratic Casimir of SU(3). Right-hand panels,(j)-(r): decomposition by the quadratic Casimir of $\operatorname{Sp}(3, R)$.

for unnatural-parity states, and has a maximum angular momentum of $13 / 2$. The identified lower band members with $J \leq 13 / 2$ all have about a $40-50 \%$ contribution from $N_{\mathrm{ex}}=1$. The band members with $J>13 / 2$ (the $15 / 2_{1}^{+}$and $17 / 2_{1}^{+}$states), which contain no $N_{\mathrm{ex}}=1$ configurations, have about $40 \%$ of their wave function in the $N_{\text {ex }}=3$ space.

The decompositions in Fig. 8 presents a clear cut contrast between $\mathrm{SU}(3)$ and $\mathrm{Sp}(3, R)$. Not surprisingly, as with our prior beryllium cases, we observe a strong difference between $\mathrm{SU}(3)$ decompositions within and without the maximal angular momentum, in Fig. 8 (h),(i). $\mathrm{Sp}(3, R)$ reveals a nearly perfect dynamical symmetry throughout the entire lower band, including the $15 / 2_{1}^{+}$and $17 / 2_{1}^{+}$states.

While we see strong transitions to an upper band in Fig. 7, the situation is nonetheless more complicated than we found in ${ }^{7,8} \mathrm{Be}$, driven at least in part, we believe, by mixing caused by the high density of states. For example, there are strong $\Delta J=2 E 2$ transitions between the $17 / 2_{1}^{+}$state and at least four $13 / 2^{+}$states; these states, the $13 / 2_{5}^{+}, 13 / 2_{6}^{+}, 13 / 2_{7}^{+}$and $13 / 2_{8}^{+}$states, which in turn have strong $E 2$ transitions to the $9 / 2_{1}$, along with strong transitions from the $13 / 2_{6}^{+}$state to the $9 / 2_{22}^{+}$state, and from $13 / 2_{7}^{+}$to $9 / 2_{18}^{+}$. The high density of states made it impractical to search for members of the band with $J<9 / 2$.

Our decompositions, shown in Fig. 9, provide additional evidence for strong mixing. (The decomposed states correspond to filled squares in Fig. 9.) While ${ }^{7,8} \mathrm{Be}$ showed rather clean decompositions, ${ }^{9} \mathrm{Be}$ shows much more fragmented structures. 


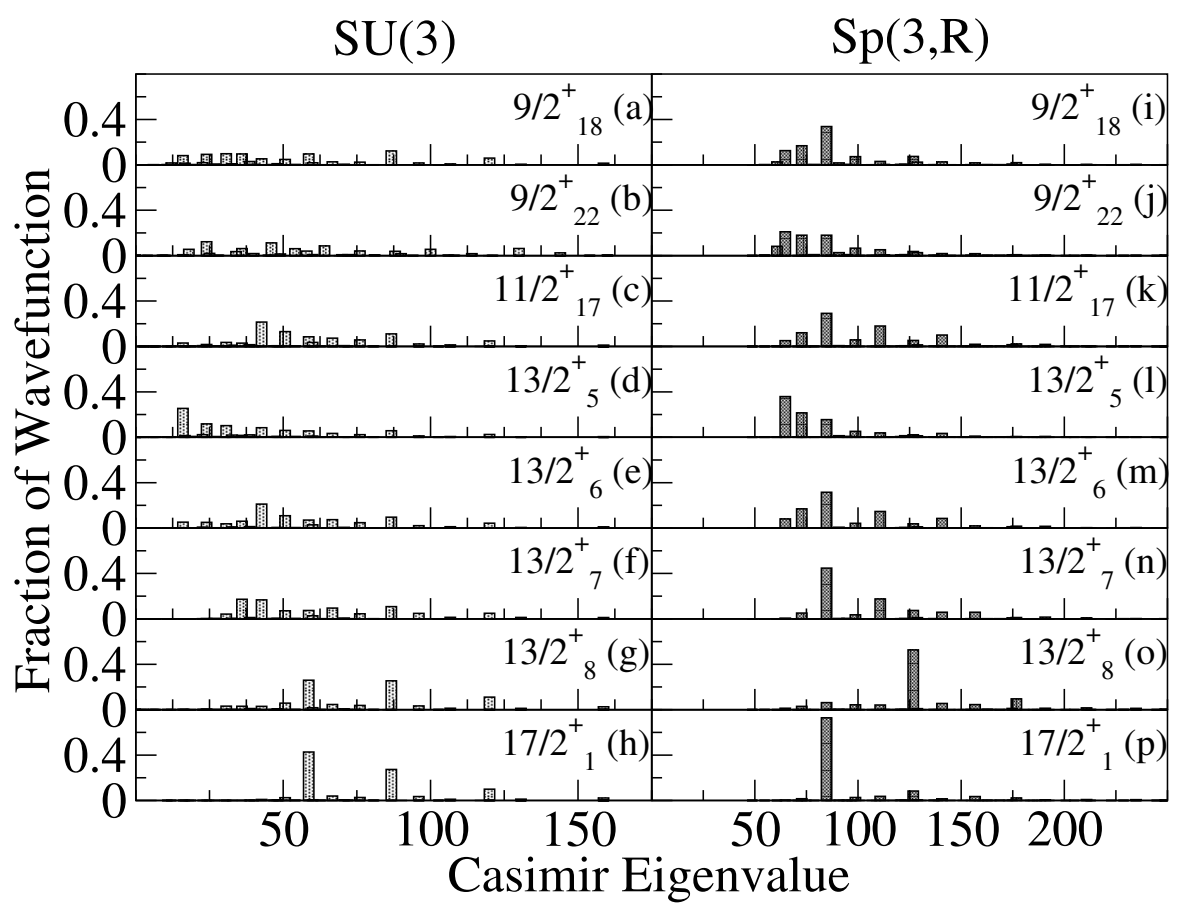

Figure 9: Decomposition of the upper rotational band members of ${ }^{9} \mathrm{Be}$ found in Fig. 7. Left-hand panels, (a)-(h): Decomposition by the quadratic Casimir of SU(3). Right-hand panels, (j)-(p): decomposition by the quadratic Casimir of $\mathrm{Sp}(3, R)$. Decompositions in panels (h),(p) are the same as Fig. 8(i),(r), added here for comparison.

The SU(3) decompositions, Fig. 9(a)-(g), in particular are highly fragmented and have little overlap with the SU(3) decomposition of the lower band in Fig. 8(a)-(i).

The $\operatorname{Sp}(3, R)$ decompositions of these excited states, Fig. $9(\mathrm{k})-(\mathrm{n})$, are less fragmented than their $\mathrm{SU}(3)$ counterparts, yet are still much more fragmented than our previous $\operatorname{Sp}(3, R)$ decompositions. The dominant $\operatorname{Sp}(3, R)$ component found in the lower band members is either dominant or subdominant in all of these upper band states, with the exception of the $13 / 2_{8}^{+}$state. Curiously, for the $13 / 2_{8}^{+}$state, the $\mathrm{SU}(3)$ decomposition matches those of the $15 / 2_{1}^{+}$and $17 / 2_{1}^{+}$states; all three of these states are predominantly $N_{\mathrm{ex}}=3$ and 5 . To aid comparison, we duplicate the decompositions of the $17 / 2_{1}^{+}$state from Fig. 8(i),(r) in Fig. 9(h),(p).

To summarize this section, ${ }^{9} \mathrm{Be}$ provides an interesting variation on our other examples. Here the $N_{\mathrm{ex}}=1$ or $1 \hbar \omega$ space functions like a valence subspace for the unnatural-parity states. The $N_{\mathrm{ex}}=1$ space also has a maximally allowed angular momentum, and states with angular momentum $J$ greater than that maximally allowed value have components with $N_{\mathrm{ex}} \geq 3$. As one follows the unnatural parity rotational band to larger angular momentum, the $\mathrm{SU}(3)$ decomposition changes as the states exit the $N_{\text {ex }}=1$ space. But, similar to other cases, the $\operatorname{Sp}(3, R)$ decompositions along the lower band remain nearly identical. While we find strong $E 2$ transitions to high-lying states reminiscent of the upper bands found in ${ }^{7,8} \mathrm{Be}$, unlike ${ }^{7,8} \mathrm{Be}$ we find much more fragmented decompositions of these states. Most of these states have a 
significant contribution from the dominant symplectic irrep of the lower band, with the exception of the $13 / 2_{8}^{+}$state.

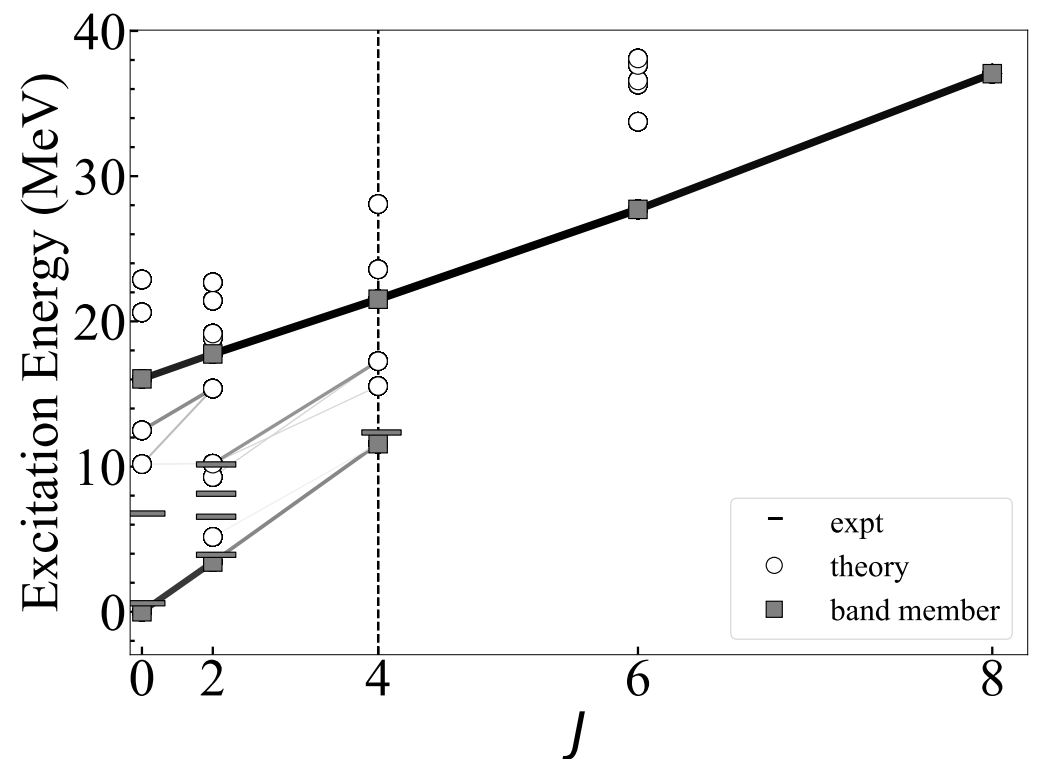

Figure 10: Excitation spectrum of the natural, positive parity states of ${ }^{10} \mathrm{Be}$, computed at $N_{\max }=8$ with a single-particle harmonic oscillator basis frequency $\hbar \omega=20 \mathrm{MeV}$, using a two-body chiral force at N3LO. Experimental energies are presented as shaded bars for comparison (see text). Rotational band members are indicated using shaded squares. These states are decomposed into group irreps in Fig. 11. Line thickness and shading is proportional to $B(E 2)$ strength. Only strengths above $0.2 e^{2} \mathrm{fm}^{4}$ are shown. The vertical dashed line denotes the end of the maximum angular momentum which can be constructed in the $N_{\text {ex }}=0$ space.

\section{4. ${ }^{10} \mathrm{Be}$}

One can think of ${ }^{10} \mathrm{Be}$ as two $\alpha$ clusters with two valence neutrons in molecular orbitals $[82,83]$. Experimentally, ${ }^{10}$ Be contains two distinct $K=0$ rotational bands [84-86], which are also expected from, e.g., antisymmetrized molecular dynamics [82] and NCSM calculations [20,39-41]. In the cluster picture, the molecular orbitals for the valence neutrons can be loosely related either to spherical shell model $p$-shell orbitals for the ground-state band, or $s d$-shell orbitals for the excited band. In the NCSM, it is therefore natural that these bands are built predominantly from $N_{\text {ex }}=0$ and $N_{\text {ex }}=2$ many-body configurations, respectively [41].

In Fig. 10 we give our $N_{\max }=8$ calculation of the rotational spectra, along with known experimental levels [69]. In addition to an yrast band consisting of only three states $\left(0_{1}^{+}, 2_{1}^{+}, 4_{1}^{+}\right)$, there is also an excited band with the $0_{4}^{+}$state as band head, which extends beyond the maximal angular momentum $J=4$ in the valence subspace. 


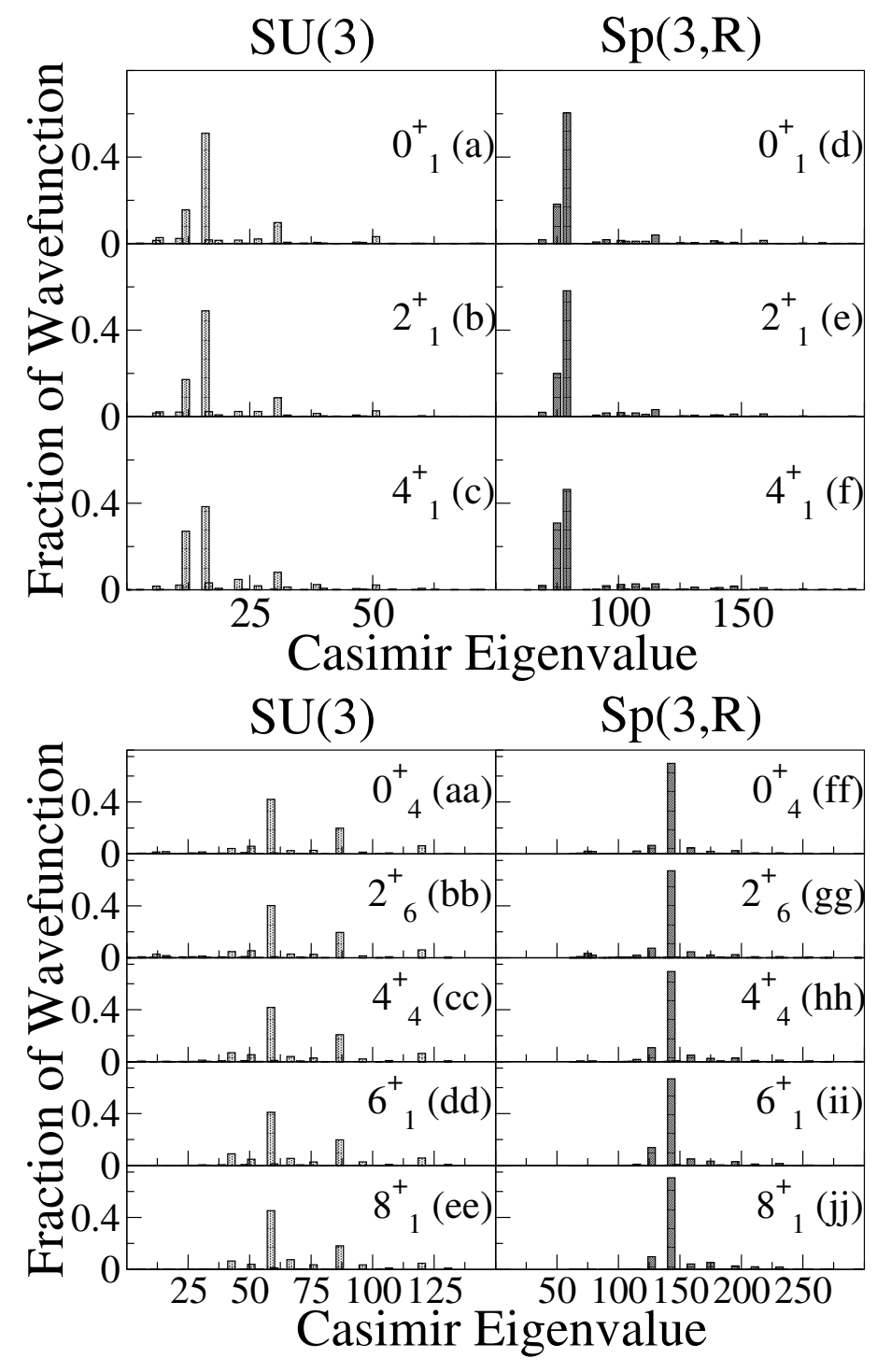

Figure 11: Group-theoretical decompositions by the quadratic Casimir of SU(3) and $\mathrm{Sp}(3, R)$ of ${ }^{10} \mathrm{Be}$ band members as calculated in Fig. 10. ground-state decompositions are shown in panels (a)-(f). Excited band decompositions are shown in panels (aa)-(jj).

Strong $E 2$ transitions link the $6_{1}^{+}, 8_{1}^{+}$not to the ground-state band but to the excited band.

This observation is supported by our analysis. Fig. 11(a)-(f) decomposes the ground-state band. As all of these have $\sim 70 \%$ of their wave functions in the $N_{\text {ex }}=0$ valence subspace, it is not surprising that they have consistent $\mathrm{SU}(3)$ and $\mathrm{Sp}(3 R)$ decompositions. The decompositions of the excited band, including the yrast $6_{1}^{+}, 8_{1}^{+}$, in Fig. 11(aa)-(jj), are markedly different. Even the members of the excited band with $J \leq 4$ have valence subspace fraction $\leq 5 \%$; instead, they consist primarily of $N_{\mathrm{ex}}=2$ 
configurations. Both bands exhibit clear evidence of quasi-dynamical symmetry, and given that the ground-state band is primarily within the valence subspace, and the excited band nearly exclusively outside the valence subspace, it is unsurprising that both $\mathrm{SU}(3)$ and $\mathrm{Sp}(3 R)$ are good symmetries.

A similar structure involving a ground-state band terminating at low $J$ and an excited band terminating at higher $J$ arises in NCSM calculations for ${ }^{11} \mathrm{Be}[20,39,42]$, with likely counterparts in experiment $[87,88]$. While we also carried out symmetry decompositions of calculations for ${ }^{11} \mathrm{Be}$, the decompositions and resulting conclusions are comparable to those shown here for ${ }^{10} \mathrm{Be}$. Indeed, they are sufficiently similar that a detailed presentation of the results would be of little further illustrative value.

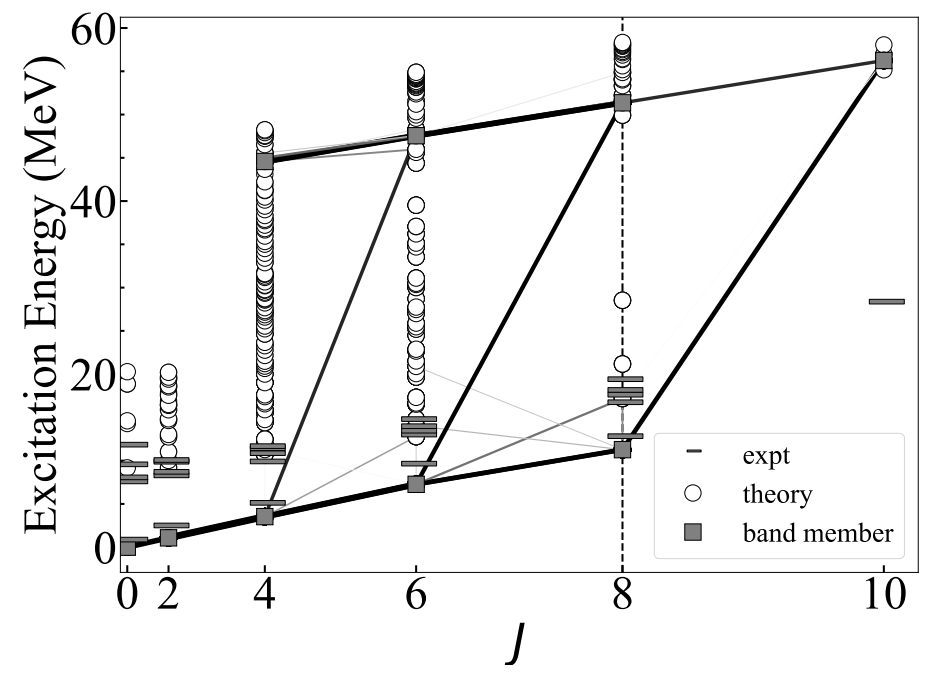

Figure 12: Excitation spectrum of the natural, positive parity states of ${ }^{20} \mathrm{Ne}$, restricted to even- $J$ states (see text), computed at $N_{\max }=4$ with a single-particle harmonic oscillator basis frequency $\hbar \omega=20 \mathrm{MeV}$, using a two-body chiral force at N3LO. Experimental energies are presented as shaded bars for comparison (see text). Rotational band members are indicated using shaded squares. These states are decomposed into group irreps in Fig. 13. J-decreasing E2 transitions between band members are indicated with solid connecting lines. Line thickness and shading is proportional to $B(E 2)$ strength. Only strengths above $0.5 e^{2} \mathrm{fm}^{4}$ are shown. The vertical dashed line denotes the maximal angular momentum within the lowest harmonic oscillator configuration.

\section{5. ${ }^{20} \mathrm{Ne}$}

We finish with ${ }^{20} \mathrm{Ne}$, known to have an yrast rotational band well-described by algebraic models [4]. This case provides an opportunity to test the pervasiveness of our findings by moving from the $p$ shell as valence subspace to the $s d$ shell. We were also motivated by a previous study [89] which found remarkable agreement, between empirical valence subspace and ab intio multi-shell calculations, in the $\mathrm{SU}(3)$ decomposition of both the ground-state and the first excited rotational bands. 
In early calculations the particle-hole structure of low-lying states was a subject of debate [90,91]; furthermore, while some particle-hole calculations failed to find strong evidence for an extended band [17] [that is, strong $B(E 2)$ values from states outside the valences subspace], later schematic symplectic calculations suggested there might indeed be an extended band $[18,19]$.

We carried out calculations at $N_{\max }=4, \ddagger$ for which the excitation spectrum and strong $E 2$ transitions are shown in Fig. 12. The maximal angular momentum for ${ }^{20} \mathrm{Ne}$ in the $s d$ shell is $J=8$. In Fig. 12 we show a ground-state band consisting of states with $J \leq 8$. However, the extra-valence $10_{2}^{+}$state appears connected to the yrast band via its strong $E 2$ transition to the $8_{1}^{+}$state.

We also observe similar strong $B(E 2)$ values from the $4_{98}^{+}, 6_{38}^{+}$, and $8_{6}^{+}$states to ground-state band members, as well as strong transitions among these states indicative of band structure. (We emphasize again that the ordering of states is specific to this model space and interaction; we do not claim, for example, that the physical $4_{98}^{+}$ state is a member of the band, only that there exists such a state. Furthermore, we expect that our calculated excitation energies are far from converged.) There are no suggestions in the calculations of any strong $E 2$ transitions from these states to any candidate odd- $J$ band members; consequently, for simplicity, only even- $J$ states are shown in Fig. 12. Thus, the excited band is identified as a $K=0$ band, even though, due to computational limitations, we are unable extend the excited band downward in angular momentum to $J=0$ and 2 .

Members of the yrast band $\left(0_{1}^{+}, 2_{1}^{+}, 4_{1}^{+}, 6_{1}^{+}, 8_{1}^{+}\right)$have approximately $75 \%$ of their wave function in the $N_{\mathrm{ex}}=0$ space, while the states we identified as belonging to the upper band, $4_{98}^{+}, 6_{38}^{+}, 8_{6}^{+}$, and $10_{2}^{+}$, have approximately $70-80 \%$ in the $N_{\mathrm{ex}}=2$ space. For yrast band members with $J \leq 8$, the $\mathrm{SU}(3)$ decompositions in Fig. 13(a)-(e) and symplectic decompositions in Fig. $13(\mathrm{~g})-(\mathrm{k})$ stay fairly consistent in appearance as they are all dominated by $N_{\mathrm{ex}}=0$ configurations. Nonetheless, we see modest evolution for decompositions with both Casimirs as $J$ increases, especially for the $6_{1}^{+}$, and $8_{1}^{+}$states in Fig. 13(d)-(e) and Fig. 13(j)-(k). Prior work suggested the $6_{1}^{+}$, and $8_{1}^{+}$states have a significantly different $N_{\text {ex }}$ fractions compared to lower angular momentum band states [17]. As with our beryllium calculations, Fig. 13(f) shows a radical change in the $\mathrm{SU}(3)$ decomposition as we go to $J=10$, where we must have $N_{\text {ex }}>0$.

By comparing the $\operatorname{Sp}(3, R)$ decompositions for the lower band in Fig. 13(g)-(l) with those for the upper band in Fig. 13(dd)-(ff), one can see ${ }^{20} \mathrm{Ne}$ has an upper band sharing the same symplectic structure. We were not expecting such a clear connection: Fig. 6(ee) and Fig. 9(h)-(n) for the beryllium examples demonstrate the $\operatorname{Sp}(3, R)$ group decomposition can fragment for highly-excited states, which we attribute to mixing with states nearby in energy. Here, for ${ }^{20} \mathrm{Ne}$, however, despite a high density of states the quasi-dynamical symmetry is nonetheless clear. Likewise, the $\mathrm{SU}(3)$ decompositions of $4_{98}^{+}, 6_{38}^{+}$, and $8_{6}^{+}$as seen in Fig. 13(aa)-(cc) are strikingly similar to one another and to the $10_{2}^{+}$state [Fig. 13(f)]. This is not surprising, as they are all dominated by $N_{\mathrm{ex}}=2$.

${ }^{20} \mathrm{Ne}$ provides strong evidence that the symplectic two-band structure found in

$\ddagger$ An $N_{\max }=4, M=0$ calculation has a dimension of approximately 75 million, while an $N_{\max }=6$, $M=0$ calculation would have a dimension of 4.4 billion. To check the need for such a large calculation, we calculated the $J=10$ states at $N_{\max }=6$, using $M=10$ which has a more manageable dimension of roughly 262 million. We found no significant difference between $N_{\max }=4$ and 6 for the SU(3) and $\operatorname{Sp}(3, R)$ decompositions of the $10_{2}^{+}$state. 


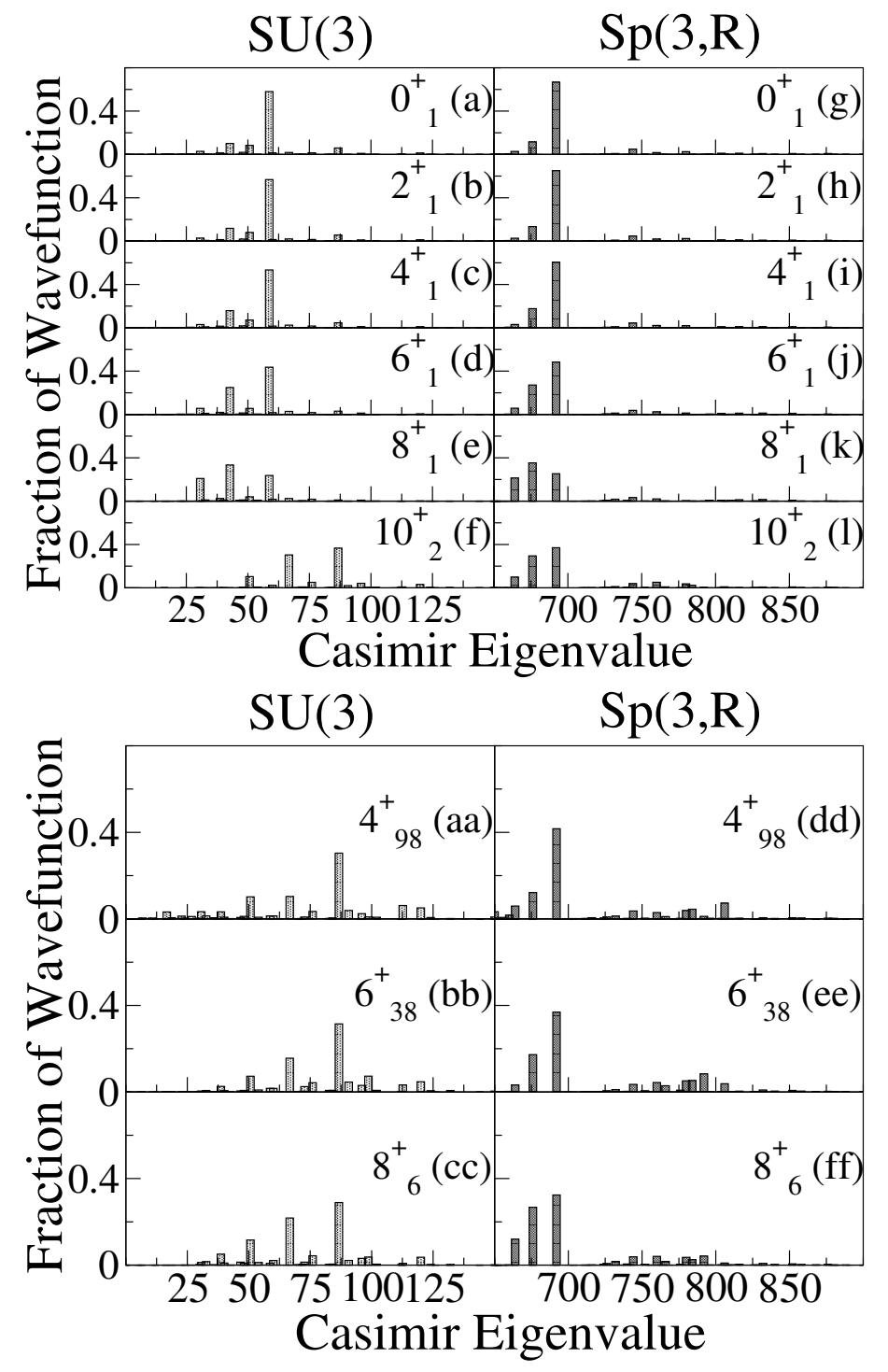

Figure 13: Group-theoretical decompositions by the quadratic Casimir of SU(3) and $\mathrm{Sp}(3, R)$ of ${ }^{20} \mathrm{Ne}$ band members as calculated in Fig. 5. Ground-state decompositions are shown in panels (a)-(l). Excited band decompositions are shown in panels (aa)-(ff).

beryllium nuclides extends to higher $A$. As methods and computing power continue to grow, it will be interesting to see how far this persists.

\section{Conclusions}

Rotational bands, identified empirically through excitation energies and strong E2 transitions, are commonplace in atomic nuclei and have a long history of being modeled algebraically and described in the context of dynamical symmetries. Of 
these symmetry frameworks, Elliott's SU(3) is the most prominent, in part because it is designed to fit neatly into restricted valence model spaces. But extended bands, that is, rotational bands that cannot be (or are not mostly) contained within the valence subspace (for example, having an angular momentum larger than is possible within the valence subspace), fall outside the purview of Elliott's SU(3) model. Making no assumptions of the underlying group structure, we decomposed no-core shell-model nuclear wave functions into subspaces related to the irrep labels, specifically, defined by $\mathrm{SU}(3)$ and $\mathrm{Sp}(3, R)$ quadratic Casimirs. Such decompositions allow one to visually compare similarities and differences in the structure of these wave functions using different algebraic lenses.

While valence subspace members of the ground-state bands follow expectations, that is, have similar $\mathrm{SU}(3)$ decompositions, those decompositions differ dramatically from those of members of the extended band. By contrast, members of an identified rotational band tend to have very similar $\operatorname{Sp}(3, R)$ decompositions.

Following previous work [33,34], we found in addition to the usual ground state or lower band a characteristic upper band that appears to unite with the lower band at high angular momentum; indeed, we view these states as part of a unified feature rather than as physically distinct bands. The upper and lower bands have the same symplectic decomposition, distinct from decomposition of other nearby excited states. This story is not simple. We did not find an upper band with the same symplectic decomposition in ${ }^{10} \mathrm{Be}$, only a band with entirely different $\mathrm{SU}(3)$ and $\mathrm{Sp}(3, R)$ structure, but it is possible we did not go up high enough in energy. We also considered a system outside the usual valence subspace, unnatural parity rotational bands in ${ }^{9} \mathrm{Be}$, where the $N_{\mathrm{ex}}=1$ or $1 \hbar \omega$ space plays a similar role to the valence subspace. While the lower positive parity band of ${ }^{9} \mathrm{Be}$ exhibits the same behavior as our other cases, the upper band has a much greater fragmentation of both $\mathrm{SU}(3)$ and $\operatorname{Sp}(3, R)$ decompositions.

Nonetheless, we also found a unified symplectic two-band structure in our calculation of ${ }^{20} \mathrm{Ne}$. Because of the excitation energies involved and the fact that the generators of $\mathrm{Sp}(3, R)$ include both quadrupole and monopole raising operators, we speculate that the upper band may be related to giant quadrupole or monopole resonances. This we leave as a topic for future investigations.

\section{Acknowledgments}

This material is based upon work supported by the U.S. Department of Energy, Office of Science, Office of Nuclear Physics, under Award Numbers DE-FG02-00ER41132, DE-FG02-03ER41272, and DE-FG02-95ER-40934. This research used computational resources of the National Energy Research Scientific Computing Center (NERSC), a U.S. Department of Energy, Office of Science, user facility supported under Contract DE-AC02-05CH11231. TRIUMF receives federal funding via a contribution agreement with the National Research Council of Canada.

\section{Appendix A. Robustness of results}

Typically in NCSM calculations, one studies the convergence as a function of the model space truncation $N_{\max }$ and of the basis oscillator frequence $\hbar \omega$. To address the question of whether or not such a study is crucial for our purposes, we investigated the robustness of our decomposition results, specifically for ${ }^{7} \mathrm{Be}$. In Fig. A1 we present 


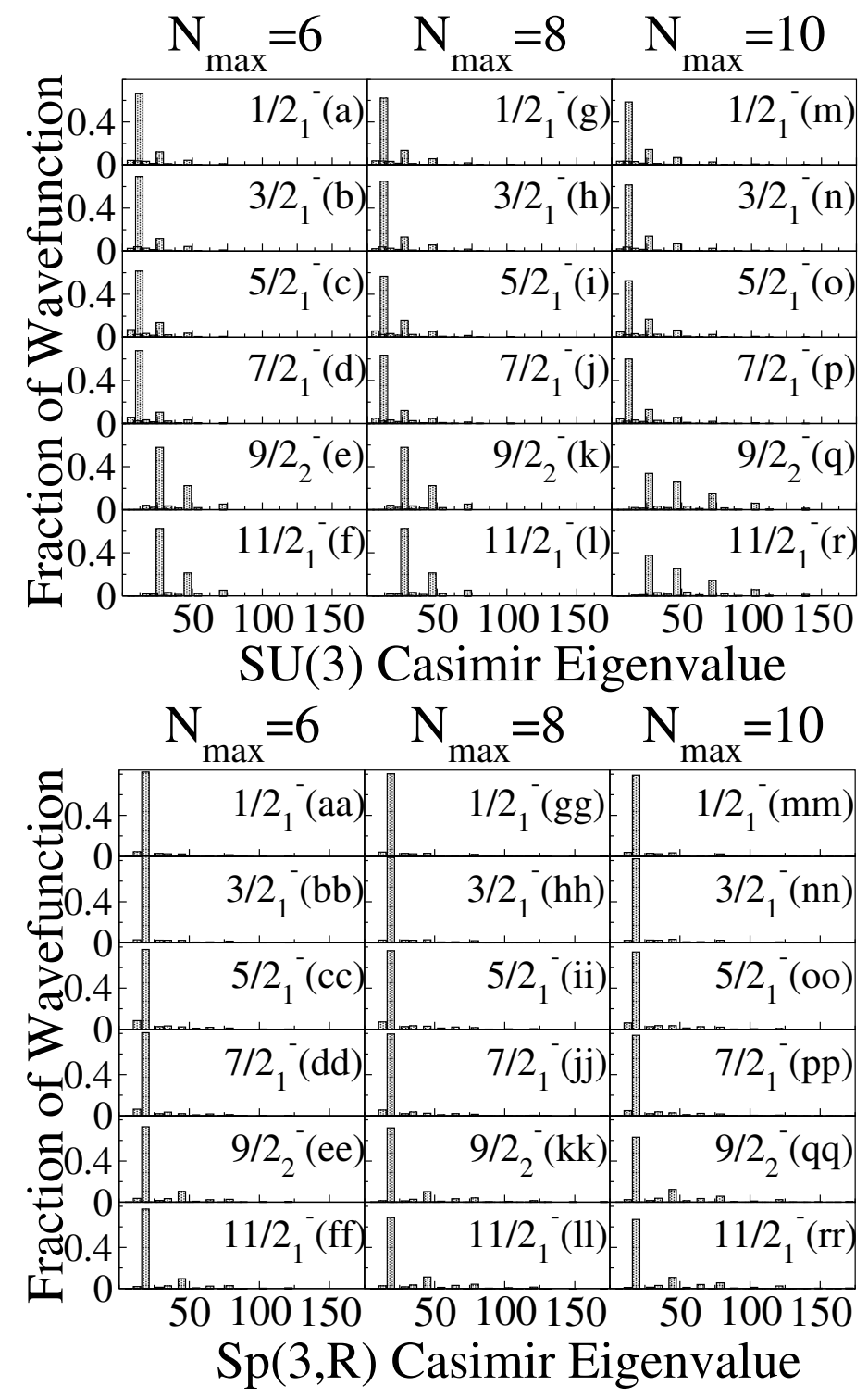

Figure A1: Decompositions of the ground-state band of ${ }^{7} \mathrm{Be}$ as calculated in Fig. 1. Panels (a)-(r): Decomposition by the quadratic Casimir of SU(3) with different $N_{\max }$ truncations. Panels (aa)-(rr): Decomposition by the quadratic Casimir of $\operatorname{Sp}(3, R)$ with different $N_{\max }$ truncations.

decompositions of the ground-state band of ${ }^{7} \mathrm{Be}$ for $N_{\max }=6,8,10$, while in Fig. A2 we decompose the $3 / 2_{1}^{-}$ground state and the excited $11 / 2_{2}^{-}$state of ${ }^{7} \mathrm{Be}$, for basis $\hbar \omega$ ranging from 12.5 to $25 \mathrm{MeV}$. The definitions of the $\mathrm{SU}(3)$ and $\mathrm{Sp}(3, R)$ generators, and thus Casimir operators, depend upon a choice for the harmonic oscillator length parameter $b$ (and thus, equivalently, $\hbar \omega$ ), as may be seen for $\hat{\mathcal{Q}}$ in (3) (see, e.g., appendix A of reference [46] for detailed definitions). The symmetry decompositions 
by use of the Casimir operator may therefore depend upon this choice as well. In all present calculations, the generators and thus the Casimirs are defined using the same $\hbar \omega$ as the NCSM oscillator basis.

In both cases, that is, varying both $N_{\max }$ (Fig. A1) and $\hbar \omega$ (Fig. A2), the decompositions are remarkably robust, with slight evolution primarily in the $\mathrm{SU}(3)$ decomposition, and most strongly in members of the extended band. These extravalence states spread across $\mathrm{SU}(3)$ irreps as $N_{\text {max }}$ increases, which accords with our understanding of these states as being slower to converge than states that reside largely within the valence space. In addition we see modest if understandable evolution with $\hbar \omega$ : if a state were, for example, wholly contained within the valence space at a specific basis frequency, changing $\hbar \omega$ would mix single-particle states with those in higher oscillator shells (in particular, states of the same angular momentum quantum numbers $l$ and $j$, but different nodal quantum numbers $n$ ), thereby changing the $N_{\text {ex }}$ and thus $\mathrm{SU}(3)$ decompositions. Nonetheless, the decomposition results are remarkable consistent, that is, largely insensitive to the details of the model space.

\section{References}

[1] Bohr A and Mottelson B R 1998 Nuclear structure vol 2 (World Scientific, Singapore)

[2] Ring P and Schuck P 2004 The nuclear many-body problem (Springer Science \& Business Media, Berlin)

[3] Rowe D J 2010 Nuclear collective motion: models and theory (World Scientific, Singapore)

[4] Rosensteel G and Rowe D 1977 Annals of Physics 104 134-144

[5] Rowe D 1996 Progress in Particle and Nuclear Physics 37 265-348

[6] Ui H 1970 Progress of Theoretical Physics 44 153-171

[7] Rosensteel G and Rowe D 1979 Annals of Physics 123 36-60

[8] Rowe D J and Wood J L 2010 Fundamentals of nuclear models: Foundational models (World Scientific, Singapore)

[9] Talmi I 1993 Simple models of complex nuclei (CRC Press)

[10] Van Isacker P 2011 Nuclear Physics A 850 157-166

[11] Elliott J P 1958 Proc. R. Soc. London A 245128

[12] Elliott J P 1958 Proc. R. Soc. London A 245562

[13] Elliott J P and Harvey M 1963 Proc. R. Soc. London A 272557

[14] Elliott J P and Wilsdon C E 1968 Proceedings of the Royal Society of London A: Mathematical, Physical and Engineering Sciences A $\mathbf{3 0 2} 509$

[15] Harvey M 1968 The nuclear SU(3) model Advances in nuclear physics (Springer, Berlin) pp $67-182$

[16] Carvalho J, Le Blanc R, Vassanji M, Rowe D and McGrory J 1986 Nuclear Physics A 452 $240-262$

[17] Goode P and Wong S 1970 Physics Letters B 32 89-91

[18] Rosensteel G and Rowe D J 1980 Ann. Phys. 126 343-370

[19] Draayer J, Weeks K and Rosensteel G 1984 Nuclear physics. A, Nuclear and hadronic physics $413215-222$

[20] Maris P, Caprio M A and Vary J P 2015 Phys. Rev. C 91(1) 014310 URL http://link.aps.org/doi/10.1103/PhysRevC.91.014310

[21] Rosensteel G and Rowe D J 1977 Phys. Rev. Lett. 38(1) 10-14 URL https://link.aps.org/doi/10.1103/PhysRevLett.38.10

[22] Dytrych T, Sviratcheva K D, Bahri C, Draayer J P and Vary J P 2007 Phys. Rev. Lett. 98(16) 162503 URL http://link.aps.org/doi/10.1103/PhysRevLett.98.162503

[23] Dytrych T, Sviratcheva K D, Bahri C, Draayer J P and Vary J P 2007 Phys. Rev. C 76(1) 014315 URL http://link.aps.org/doi/10.1103/PhysRevC.76.014315

[24] Dytrych T, Sviratcheva K D, Draayer J P, Bahri C and Vary J P 2008 Journal of Physics G: Nuclear and Particle Physics 35123101 URL http://stacks.iop.org/0954-3899/35/i=12/a=123101

[25] Dytrych T, Sviratcheva K D, Bahri C, Draayer J P and Vary J P 2008 Journal of Physics G: Nuclear and Particle Physics 35095101 URL http://stacks.iop.org/0954-3899/35/i=9/a=095101 


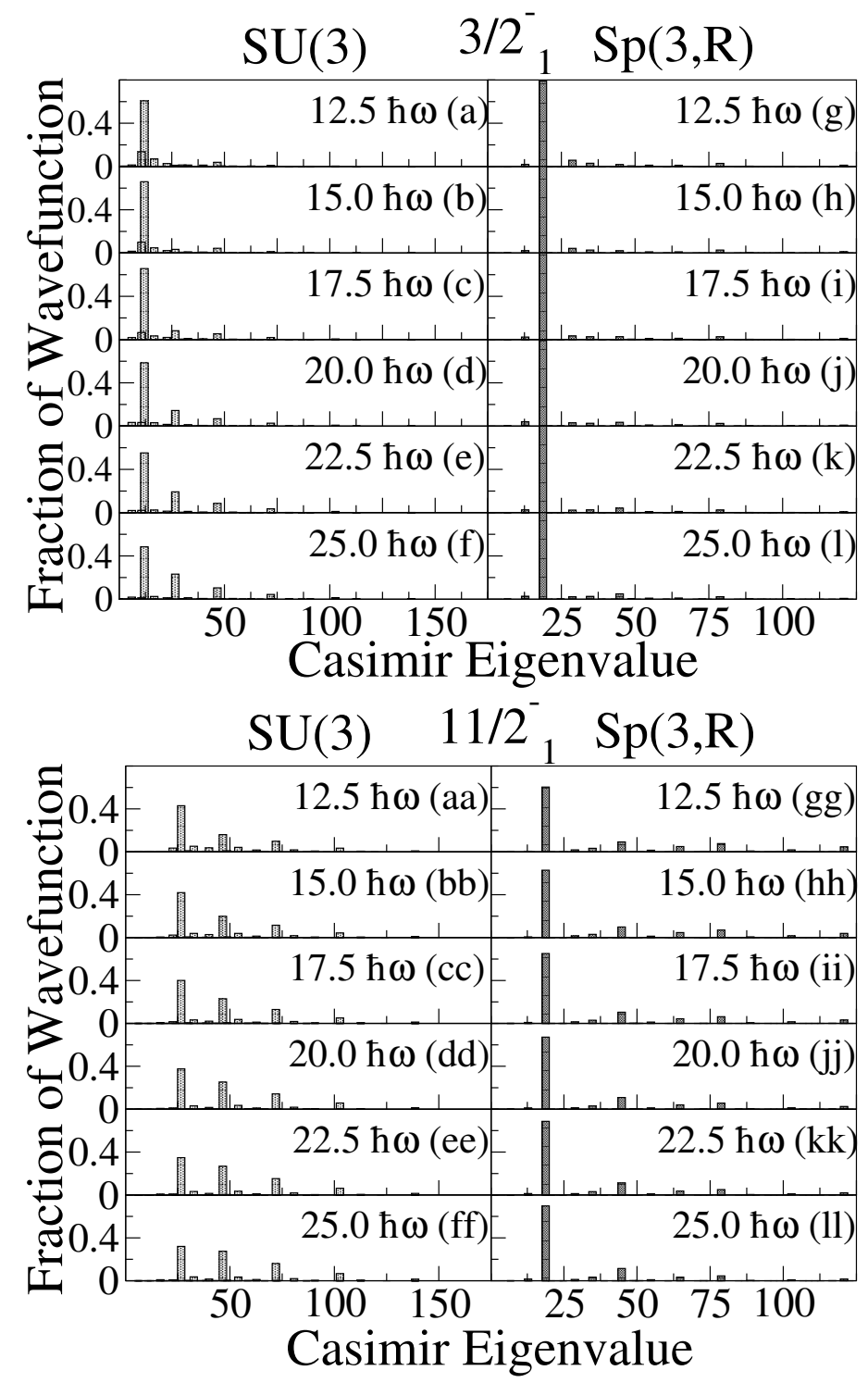

Figure A2: Group-theoretical $\mathrm{SU}(3)$ and $\operatorname{Sp}(3, R)$ decompositions of the $J=3 / 2^{-}$ ground state, and first excited $J=11 / 2^{-}$state of ${ }^{7} \mathrm{Be}$ computed at $N_{\max }=10 \mathrm{using}$ a two-body chiral force at N3LO interaction. Each decomposition panel is labeled by the single-particle harmonic oscillator basis frequency value used to create it. Groundstate decompositions are shown in panels (a)-(l). Decompositions of the $11 / 2_{1}^{-}$state are shown in panels (aa)-(ll).

[26] Draayer J P, Dytrych T, Launey K D and Langr D 2012 Prog. Part. and Nucl. Phys. 67516

[27] Dytrych T, Launey K D, Draayer J P, Maris P, Vary J P, Saule E, Catalyurek U, Sosonkina M, Langr D and Caprio M A 2013 Phys. Rev. Lett. 111(25) 252501 URL http://link.aps.org/doi/10.1103/PhysRevLett.111.252501

[28] Dytrych T, Maris P, Launey K D, Draayer J P, Vary J P, Langr D, Saule E, Caprio M, Catalyurek 
U and Sosonkina M 2016 Computer Physics Communications 207 202-210

[29] Launey K D, Dytrych T and Draayer J P 2016 Progress in Particle and Nuclear Physics 89 $101-136$

[30] Dytrych T, Launey K D, Draayer J P, Rowe D J, Wood J L, Rosensteel G, Bahri C, Langr D and Baker R B 2020 Phys. Rev. Lett. 124042501

[31] Draayer J, Dytrych T and Launey K 2011 Ab initio symmetry-adapted no-core shell model Journal of Physics: Conference Series vol 322 (IOP Publishing) p 012001

[32] McCoy A E, Caprio M A and Dytrych T 2018 Ann. Acad. Rom. Sci. Ser. Chem. Phys. Sci. 3 17

[33] McCoy A E 2018 Ab initio multi-irrep symplectic no-core configuration interaction calculations Ph.D. thesis University of Notre Dame URL https://curate.nd.edu/show/pz50gt57p16

[34] McCoy A E, Caprio M A, Dytrych T and Fasano P J 2020 Phys. Rev. Lett. 125(10) 102505 URL https://link.aps.org/doi/10.1103/PhysRevLett.125.102505

[35] Machleidt R and Entem D R 2011 Physics Reports 503 1-75

[36] Gueorguiev V G, Draayer J P and Johnson C W 2000 Phys. Rev. C 63(1) 014318 URL http://link.aps.org/doi/10.1103/PhysRevC.63.014318

[37] Johnson $\mathrm{C} \quad \mathrm{W} \quad 2015$ Phys. Rev. $C \quad$ 91(3) 034313 URL http://link.aps.org/doi/10.1103/PhysRevC.91.034313

[38] Herrera R A and Johnson C W 2017 Phys. Rev. C 95(2) 024303 URL https://link.aps.org/doi/10.1103/PhysRevC.95.024303

[39] Caprio M A, Maris P and Vary J P 2013 Physics Letters B 719 179-184

[40] Caprio M A, Maris P, Vary J P and Smith R 2015 International Journal of Modern Physics E 241541002

[41] Caprio M A, Fasano P J, McCoy A E, Maris P and Vary J P 2019 Bulg. J. Phys. 46445 URL https: //www . bjp-bg.com/paper.php?id=1208

[42] Caprio M A, Fasano P J, Maris P, McCoy A E and Vary J P 2020 The European Physical Journal A 56 1-20

[43] Brussard P and Glaudemans P 1977 Shell-model applications in nuclear spectroscopy (NorthHolland Publishing Company, Amsterdam)

[44] Brown B A and Wildenthal B H 1988 Annual Review of Nuclear and Particle Science 38 29-66

[45] Caurier E, Martinez-Pinedo G, Nowacki F, Poves A and Zuker A P 2005 Reviews of Modern Physics $\mathbf{7 7}(2)$ 427-488

[46] Caprio M A, McCoy A E and Fasano P J 2020 J. Phys. G 47122001

[47] Navrátil P, Vary J and Barrett B 2000 Physical Review C 62054311

[48] Barrett B R, Navrátil P and Vary J P 2013 Progress in Particle and Nuclear Physics 69 131-181

[49] Bogner S K, Furnstahl R J and Perry R J 2007 Phys. Rev. C 75(6) 061001 URL http://link.aps.org/doi/10.1103/PhysRevC.75.061001

[50] Johnson C W, Ormand W E and Krastev P G 2013 Computer Physics Communications 184 $2761-2774$

[51] Johnson C W, Ormand W E, McElvain K S and Shan H 2018 arXiv preprint arXiv:1801.08432

[52] Whitehead R R, Watt A, Cole B J and Morrison I 1977 Adv. Nuclear Phys. Vol. 9, pp 123-176

[53] Hecht K T 1973 Annu. Rev. Nucl. Sci. 23123

[54] Wybourne B G 1974 Classical Groups for Physicists (New York: Wiley)

[55] Chen J Q, Ping J and Wang F 1989 Group representation theory for physicists vol 7 (World Scientific)

[56] Iachello F 1994 Algebraic theory Lie Algebras, Cohomology, and New Applications to Quantum Mechanics (Contemp. Math. vol 160) ed Kamran N and Olver P (Providence, Rhode Island: American Mathematical Society) p 151

[57] Iachello F 2015 Lie Algebras and Applications 2nd ed (Lecture Notes in Physics vol 891) (Berlin: Springer)

[58] Rowe D J, McCoy A E and Caprio M A 2016 Physica Scripta 91033003

[59] Rochford P and Rowe D 1988 Physics Letters B 210 5-9

[60] Bahri C, Rowe D J and Wijesundera W 1998 Phys. Rev. C 58(3) 1539-1550 URL http://link.aps.org/doi/10.1103/PhysRevC.58.1539

[61] Rowe D J 2000 Quasi-dynamical symmetry:a new use of symmetry in nuclear physics The Nucleus (Springer) pp 379-395

[62] Bahri C and Rowe D 2000 Nuclear Physics A 662 125-147

[63] Whitehead R R 1980 Theory and Applications of Moment Methods in Many-Fermion Systems ed Dalton B J, Grimes S M, Vary J P and Williams S A (New York: Plenum) p 235

[64] Escher J and Leviatan A 2002 Phys. Rev. C 65(5) 054309 URL https://link.aps.org/doi/10.1103/PhysRevC.65.054309 
[65] Shirokov A, Vary J, Mazur A and Weber T 2007 Physics Letters B 644 33-37

[66] Ekström A, Baardsen G, Forssén C, Hagen G, Hjorth-Jensen M, Jansen G R, Machleidt R, Nazarewicz W, Papenbrock T, Sarich J and Wild S M 2013 Phys. Rev. Lett. 110192502

[67] Shirokov A, Shin I, Kim Y, Sosonkina M, Maris P and Vary J 2016 Physics Letters B 761 87-91

[68] Tilley D, Cheves C, Godwin J, Hale G, Hofmann H, Kelley J, Sheu C and Weller H 2002 Nuclear Physics A 708 - 163 ISSN 0375-9474 URL http://www.sciencedirect.com/science/article/pii/S0375947402005973

[69] Tilley D, Kelley J, Godwin J, Millener D, Purcell J, Sheu C and Weller H 2004 Nuclear Physics A $\mathbf{7 4 5} 155$ - 362 ISSN 0375-9474 URL http://www.sciencedirect.com/science/article/pii/S0375947404010267

[70] Yoshida T, Shimizu N, Abe T and Otsuka T 2013 Cluster structure in Monte Carlo shell model Journal of Physics. Conference Series (Online) vol 454

[71] Bouten M, Bouten M, Depuydt H and Schotsmans L 1970 Physics Letters B 33 457-459

[72] Bouten M, Bouten M and Van Leuven P 1971 Nuclear Physics A 168 438-448

[73] Arickx F, Van Leuven P and Bouten M 1975 Nuclear Physics A 252 416-422

[74] Arickx F 1976 Nuclear Physics A 268 347-357

[75] Arickx F, Broeckhove J and Deumens E 1979 Nuclear Physics A 318 269-286

[76] Adler C, Corcoran T and Mast C 1966 Nuclear Physics 88 145-168

[77] Millener D 2001 Nuclear Physics. A 693 394-410

[78] Bouten M, Bouten M, Depuydt H and Schotsmans L 1968 Physics Letters B 27 61-64

[79] Okabe S, Abe Y and Tanaka H 1977 Progress of Theoretical Physics 57 866-881

[80] Forssén C, Navrátil P, Ormand W E and Caurier E 2005 Phys. Rev. C 71(4) 044312 URL https://link.aps.org/doi/10.1103/PhysRevC.71.044312

[81] Caprio M A, Fasano P J, Vary J P, Maris P and Hartley J 2019 Robust ab initio predictions for nuclear rotational structure in the Be isotopes Proceedings of the International Conference Nuclear Theory in the Supercomputing Era 2018 ed Shirokov A M and Mazur A I (Pacific National University, Khabarovsk, Russia) p 250 URL http://www.ntse.khb.ru/files/uploads/2018/proceedings/Caprio.pdf

[82] Kanada-En'yo Y, Horiuchi H and Doté A 1999 Phys. Rev. C 60064304

[83] Lashko Y A, Filippov G and Vasilevsky V 2017 Nuclear Physics A 958 78-100

[84] Freer M, Casarejos E, Achouri L, Angulo C, Ashwood N I, Curtis N, Demaret P, Harlin C, Laurent B, Milin M, Orr N A, Price D, Raabe R, Soić N and Ziman V A 2006 Phys. Rev. Lett. 96(4) 042501 URL https://link.aps.org/doi/10.1103/PhysRevLett.96.042501

[85] Bohlen H G, Dorsch T, Kokalova T, Oertzen W v, Schulz C and Wheldon C 2007 Phys. Rev. C 75(5) 054604 URL https://link.aps.org/doi/10.1103/PhysRevC.75.054604

[86] Suzuki D, Shore A, Mittig W, Kolata J J, Bazin D, Ford M, Ahn T, Becchetti F D, Beceiro Novo S, Ben Ali D, Bucher B, Browne J, Fang X, Febbraro M, Fritsch A, Galyaev E, Howard A M, Keeley N, Lynch W G, Ojaruega M, Roberts A L and Tang X D 2013 Phys. Rev. C 87(5) 054301 URL https://link.aps.org/doi/10.1103/PhysRevC.87.054301

[87] Bohlen H G, von Oertzen W, Kalpakchieva R, Massey T N, Dorsch T, Milin M, Schulz Ch, Kokalova Tz and Wheldon C 2008 J. Phys. Conf. Ser. 111012021

[88] Chen J, Auranen K, Avila M L, Back B B, Caprio M A, Hoffman C R, Gorelov D, Kay B P, Kuvin S A, Liu Q, Lou J L, Macchiavelli A O, McNeel D G, Tang T L, Santiago-Gonzalez D, Talwar R, Wu J, Wilson G, Wiringa R B, Ye Y L, Yuan C X and Zang H L 2019 Phys. Rev. C 100064314

[89] Johnson C W 2017 The anatomy of atomic nuclei: illuminating many-body wave functions through group-theoretical decomposition Emergent Phenomena In Atomic Nuclei From Large-scale Modeling: A Symmetry-guided Perspective ed Launey K D (World Scientific, Singapore) p 33

[90] Abulaffio C 1966 Nuclear Physics 81 71-75

[91] Vogt E 1972 Physics Letters B 40 345-348 